\title{
Vector Control of a Modular Multilevel Matrix Converter Operating over the Full Output-Frequency Range
}

\author{
Matias Diaz, Member, IEEE, Roberto Cardenas, Senior Member, IEEE, Mauricio Espinoza, Member, \\ IEEE, Christoph Hackl, Senior Member, IEEE, Felix Rojas, Jon Clare, Senior Member, IEEE, \\ Pat Wheeler, Senior Member, IEEE
}

\begin{abstract}
The Modular Multilevel Matrix Converter $\left(M^{3} C\right)$ is an ac-to-ac converter topology suitable for the control of high-power variable-speed drives. The control of this converter is complex, particularly when the two ac system frequencies are similar or identical because large voltage oscillations can be produced in the floating capacitors within the $M^{3} C$. This paper proposes a new Vector Control System based on nested controllers to regulate the $M^{3} C$ over the full-range of frequencies. The proposed control scheme is especially useful to mitigate or eliminate the oscillations that arise when the frequencies are similar. An extensive discussion of the model and control of the $M^{3} C$ is presented in this work. The effectiveness of the proposed Vector Control System is demonstrated through simulation studies and experimental validation tests conducted with a 27-cell-5kW $M^{3} C$ prototype.
\end{abstract}

Index Terms-ac-to-ac power conversion, Equal Frequencies Operation, Modular Multilevel Matrix Converter

\section{INTRODUCTION}

$\mathbf{M}$ ODULAR Multilevel Cascade Converters (MMCC) are a family of power converters proposed initially for High Voltage DC transmission [1]. $M M C C$ have several advantages over traditional topologies such as full modularity, simple extension to high voltage levels, redundancy, control flexibility and power quality [2]. More recently, recognising these advantages, MMCCs have been proposed for use in motor-drives for SAG mills, conveyors, mine hoists and wind turbine applications [2]-[5].

Manuscript received February 22, 2018; revised April 08, 2018; accepted August 27, 2018. This work was supported by FONDECYT grant 1180879. The funding provided by the University of Santiago of Chile through projects 091813DD and 091813DDRED is also recognised.

Matias Diaz and Felix Rojas are with the Electrical Engineering Department, the University of Santiago of Chile, Avenida Ecuador 3519, Santiago, Chile (e-mail: matias.diazd@usach.cl, felix.rojas@usach.cl),

Roberto Cardenas is with the Electrical Engineering Department, University of Chile, Avenida Tupper 2007, Santiago, Chile (e-mail: rcd@ieee.org).

Mauricio Espinoza is with the Faculty of Engineering, University of Costa Rica, San Pedro, Costa Rica (e-mail: maeb@ieee.org),

Christoph Hackl is with the Munich University of Applied Sciences (MUAS) and head of the "Control of Renewable Energy Systems (CRES)" research group at Technical University of Munich (TUM) (email: christoph.hackl@hm.edu).

Jon Clare and Pat Wheeler are with the Faculty of Engineering, University of Nottingham, Nottingham, NG7 2RD, UK. (e-mail: jon.clare@nottingham.ac.uk, pat.wheeler@nottingham.ac.uk ).

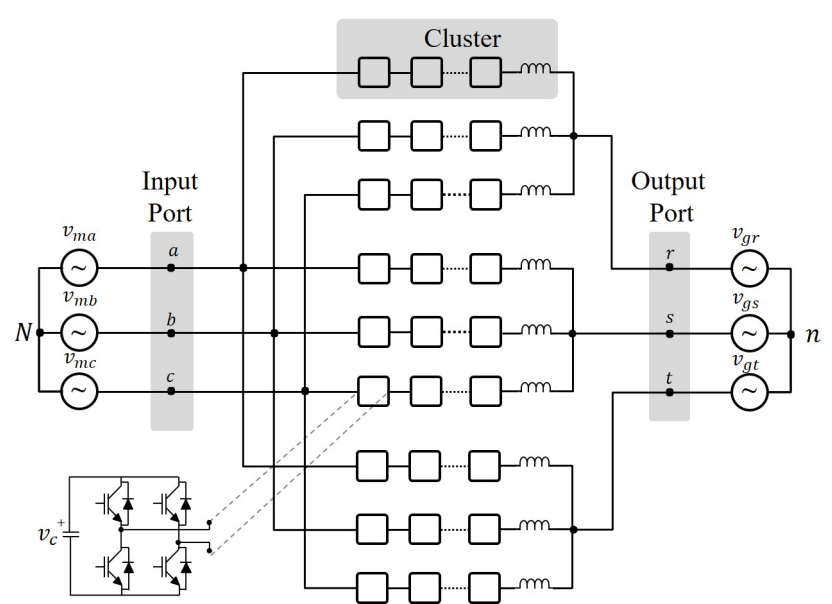

Fig. 1. Modular Multilevel Matrix Converter Topology.

The MMCC family is characterised by a cascaded connection of cells forming a cluster or arm (see Fig. 1) and the converter voltage rating can be increased by using a higher number of cells per cluster. The capacitor voltage of each cell is floating and can be charged/discharged during the operation of the converter. Therefore, one of the most important control tasks is to maintain each capacitor voltage within an acceptable range, mainly for variable speed operation [3]. Of this family, the most investigated topologies for ac-to-ac applications are the Modular Multilevel Converter $\left(M^{2} C\right)$ and the Modular Multilevel Matrix Converter $\left(M^{3} C\right)$, being the $M^{3} C$ recommended for low-speed high-power applications [2].

The $M^{3} C$ has 9 clusters based on series connected fullbridge cells allowing direct connection of two ac ports as shown Fig. 1. This converter is suitable for low-speed highpower applications because lower circulating currents are required to mitigate the capacitor voltage oscillations compared to those required in the $M^{2} C$ [6], [7]. However, the $M^{3} C$ has an inherent problem operating when the input/output frequencies are similar/identical because large capacitor voltage oscillations can be produced [7].

Recently, nested control systems based on decoupled modelling of the $M^{3} C$ have been proposed [4], [5]. It is claimed that these approaches decouple the converter voltages and currents simplifying the control of circulating currents and common-mode voltage in the $M^{3} C$. For the sake of simplicity, in this work the operating range of the $M^{3} C$ will be divided 
into two modes: the Different Frequencies Mode (DFM) and the Equal Frequencies Mode (EFM). When the input-port frequency is different to the output-port frequency (i.e. lower or higher by a given threshold), the system is considered to operate in DFM. In this zone, the capacitor voltage mean values are controlled using either circulating currents or by injecting a common-mode voltage. Some publications have reported experimental validation of DFM control systems of the $M^{3} C$ for drive applications [4], [5] and Wind Energy Conversion Systems [8]-[10] and ac-ac conversion [11]-[13]. On the other hand, when the absolute value of the input-port frequency is very close or equal to the output-port frequency, the system is considered to operate in EFM, where mitigation signals or operation point restrictions are utilised in the control systems to eliminate the oscillations in the floating capacitor voltages [14]-[18]. The average value of the capacitor voltages are regulated as in DFM, and the oscillations can be attenuated by introducing some operational restrictions such as, for instance, imposing complementary input/output power factors and equal input/output voltage amplitudes in the converter [14], [16]. Alternatively, common-mode voltages and circulating currents can be calculated offline and stored in a look-up table as part of an open-loop mitigation scheme [15], [17], [18]. However, the use of mitigation signals imposed in an open-loop manner during EFM cannot compensate for non-linearities, changes in the operating point and uncertainties in the converter model. Therefore methods based on using look-up tables cannot ensure good performance for all operating conditions. In [19], closed-loop control of the mitigation signals is proposed. However, in this preliminary work, only simulations results are presented for the case where the input-output frequencies of the converter are equal. Moreover, the performance of the proposed control methodology is not compared with that produced with the existing control systems. The contributions of this paper can be summarised as follows:

- To the best our knowledge, this is the first paper where vector control systems for the $M^{3} C$ are developed and experimentally validated for the whole operating range. The performance of the proposed control system is compared with that obtained using some of the previously reported control methodologies. The voltage oscillations are directly controlled using the proposed control system without using off-line-calculated mitigations signals stored in look-up tables.

- The proposed control systems can be applied over the whole frequency range, including DFM and EFM operation. The floating capacitor energies of the $M^{3} C$ are regulated using nested controllers, where the outer loops regulate the capacitor voltages by setting the circulating current references.

- For EFM, the mitigation signals are regulated using closed-loop controllers that successfully drive the lowfrequency oscillations, which can otherwise lead to large voltage oscillations, to zero. Therefore, the control system has all the advantages of standard vector control systems which are well discussed in the literature for field orientated control [20] and power converters [3]. This is

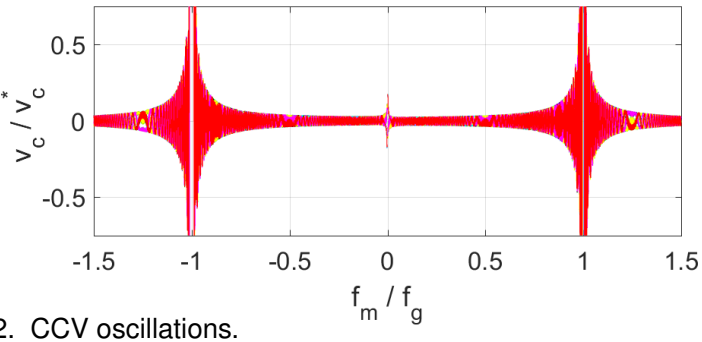

Fig. 2. CCV oscillations.

different to the control systems previously published for $M^{3} C$ applications (see [15], [17], [18]) where Proportional and PI controllers implemented in the stationary $\alpha \beta$ frame are utilised, which cannot regulate sinusoidal signals with zero steady-state error.

- The effectiveness of the proposed control system is validated through simulations and experiments conducted with a $27-$ cell-5kVA prototype.

\section{Modular Multilevel Matrix Converter}

The $M^{3} C$ can be represented by a model expressed in double $\alpha \beta 0$ frame, obtaining a Voltage-Current model and a Power-Capacitor Voltage model. The double $\alpha \beta 0$ transform has been discussed in [4], [5], [10] and it is obtained by using $\left[X_{\alpha \beta 0}\right]=[C]\left[X_{a b c}\right][C]^{t}$, where $[C]$ represents the Clarke transform and the subscript $a b c$ represents natural coordinates. The main advantage of the double $\alpha \beta 0$ transform is in decoupling the converter voltages and currents, which simplifies the design and implementation of control systems [4], [5].

\section{A. Voltage-Current Model of the $M^{3} C$}

The Voltage-Current model of the $M^{3} C$ in the double $\alpha \beta 0$ frame is given in (1) [4], [5]:

$$
\begin{aligned}
& \sqrt{3}\left[\begin{array}{ccc}
0 & 0 & 0 \\
0 & 0 & 0 \\
v_{m_{\alpha}} & v_{m_{\beta}} & 0
\end{array}\right]=L_{c} \frac{d}{d t}\left[\begin{array}{ccc}
i_{\alpha \alpha} & i_{\beta \alpha} & i_{0 \alpha} \\
i_{\alpha \beta} & i_{\beta \beta} & i_{0 \beta} \\
i_{\alpha 0} & i_{\beta 0} & i_{00}
\end{array}\right] \\
& +\left[\begin{array}{lll}
v_{\alpha \alpha} & v_{\beta \alpha} & v_{0 \alpha} \\
v_{\alpha \beta} & v_{\beta \beta} & v_{0 \beta} \\
v_{\alpha 0} & v_{\beta 0} & v_{00}
\end{array}\right]+\sqrt{3}\left[\begin{array}{ccc}
0 & 0 & v_{g_{\alpha}} \\
0 & 0 & v_{g_{\beta}} \\
0 & 0 & 0
\end{array}\right]+\left[\begin{array}{ccc}
0 & 0 & 0 \\
0 & 0 & 0 \\
0 & 0 & 3 v_{n}
\end{array}\right]
\end{aligned}
$$

Note that the currents $i_{\alpha 0}$ and $i_{\beta 0}$ are only dependent on the input-port currents, whereas $i_{0 \alpha}$ and $i_{0 \beta}$ are only dependent on the output-port currents. Furthermore, $i_{\alpha \alpha}, i_{\beta \alpha}, i_{\beta \alpha}$ and $i_{\beta \beta}$ are circulating currents which have no effect on either the input/output ports.

\section{B. Power-Capacitor Voltage Model of the $M^{3} C$}

Assuming that all the capacitor voltages are well regulated with a value close to the demanded voltage $v_{c}^{*}$, then the total capacitor voltage available in a cluster, referred to as the Cluster Capacitor Voltage (CCV), can be related to the cluster power as given in (2).

As mentioned before, the floating capacitors can charge or discharge during the operation of the converter, and at some input/output frequency operating points the control systems required to regulate these variations can be relatively complex. For instance using a simulation, the nine CCVs are plotted (in per-unit form) as a function of the input-output port difference frequency in Fig. 2. The parameters of the experimental setup 
presented in Section VI are used. In this case, the input-port frequency $\left(f_{m}\right)$ is varied from $-75 \mathrm{~Hz}$ to $75 \mathrm{~Hz}$ while the output-port frequency $\left(f_{g}\right)$ is fixed at $50 \mathrm{~Hz}$. The CCVs can have very large voltage oscillations when $f_{m}$ is close or equal to $\pm f_{g}$. In the other cases, when the absolute frequencies are disimilar, the CCV oscillations are not very large. However, due to the integrating effect of the capacitors, regulation is required over the whole speed operating range when it is considered that even small power variations can produce significant voltage imbalances.

The $M^{3} C$ Power-CCV model of the $M^{3} C$ in double $\alpha \beta 0$ frame is [4], [5]:

$$
C v_{c}^{*} \frac{d}{d t}\left[\begin{array}{lll}
v_{c_{\alpha \alpha}} & v_{c_{\beta \alpha}} & v_{c_{0 \alpha}} \\
v_{c_{\alpha \beta}} & v_{c_{\beta \beta}} & v_{c_{0 \beta}} \\
v_{c_{\alpha 0}} & v_{c_{\beta 0}} & v_{c_{00}}
\end{array}\right] \approx\left[\begin{array}{ccc}
P_{\alpha \alpha} & P_{\beta \alpha} & P_{0 \alpha} \\
P_{\alpha \beta} & P_{\beta \beta} & P_{0 \beta} \\
P_{\alpha 0} & P_{\beta 0} & P_{00}
\end{array}\right]
$$

Where the voltage terms of the left-side of (2) have the following physical meanings:

- The terms $v_{c_{\alpha \alpha}}, v_{c_{\beta \alpha}}, v_{c_{\alpha \beta}}, v_{c_{\beta \beta}}, v_{c_{0 \alpha}}, v_{c_{0 \beta}}, v_{c_{\alpha 0}}$ and $v_{c_{\beta 0}}$ represent $\mathrm{CCV}$ imbalances. The mean value of those terms tends to zero when the converter is properly regulated.

- The term $v_{c_{00}}$ is related to the total active power flowing into/from the $M^{3} C$ and can be regulated to set the average total value of the CCVs.

The power components on the right-hand side of (2) can be expressed as a function of the transformed currents and voltages as proposed in [5]. For instance, the cluster power term $P_{\alpha \alpha}$ can be expressed as:

$$
\begin{aligned}
P_{\alpha \alpha} & =\overbrace{\frac{\overbrace{\left(v_{m \alpha} i_{g \alpha}-v_{g \alpha} i_{m \alpha}\right)}^{3}}{1 \text { st component }}+\overbrace{\frac{\left(v_{m \alpha} i_{\alpha \alpha}-v_{m \beta} i_{\beta \alpha}\right)}{\sqrt{6}}}^{\text {2nd component }}}^{\text {3rd component }} \\
& -\overbrace{\frac{\left(v_{g \alpha} i_{\alpha \alpha}-v_{g \beta} i_{\alpha \alpha}\right)}{\sqrt{6}}}^{4 \text { th component }}-\overbrace{v_{n} i_{\alpha \alpha}}
\end{aligned}
$$

The complete expression of the remaining seven power components (i.e. $P_{\alpha \beta}, P_{\beta \alpha}, P_{\beta \beta}, P_{\alpha 0}, P_{\beta 0}, P_{0 \alpha}, P_{0 \beta}$ ) can be found in [5]. Assuming that neither circulating currents nor commonmode voltage are applied, it can be concluded that the oscillations in the capacitors are produced by the first component of (3). For example, using (2)-(3), the oscillating component of $v_{c_{\alpha \alpha}}$ can be approximated as:

$$
\begin{aligned}
& \tilde{v}_{c_{\alpha \alpha}} \approx \frac{V_{m} I_{g} \sin \left(\phi_{g}+\left(\omega_{g}-\omega_{m}\right) t\right)+V_{g} I_{m} \sin \left(\phi_{m}-\left(\omega_{g}-\omega_{m}\right) t\right)}{6 C v_{c}^{*}\left(\omega_{g}-\omega_{m}\right)} \\
& +\frac{V_{m} I_{g} \sin \left(\phi_{g}+\left(\omega_{g}+\omega_{m}\right) t\right)-V_{g} I_{m} \sin \left(\phi_{m}+\left(\omega_{g}+\omega_{m}\right) t\right)}{6 C v_{c}^{*}\left(\omega_{g}+\omega_{m}\right)}+\tilde{v}_{c_{\alpha \alpha}}^{h f}
\end{aligned}
$$

where $\omega_{m}=2 \pi f_{m}$ and $\omega_{g}=2 \pi f_{g}$. Note that $V_{m}$ and $V_{g}$ are the input-port and the output-port phase-to-neutral peak voltage magnitudes, respectively. $I_{m}$ and $I_{g}$ are the input-port and the output-port peak current magnitudes, respectively. $f_{m}$ and $\phi_{m}$ are the input-port frequency and phase angle, whereas $f_{g}$ and $\phi_{g}$ are output-port frequency and phase angle. The term $\tilde{v}_{c_{\alpha \alpha}}^{h f}$ also has components of frequencies $f_{g}$ and $f_{m}$

The term $v_{c_{\alpha \alpha}}$ can present theoretically unbounded voltage oscillations when $f_{m}= \pm f_{g}$. In this condition, the same problem appears in $v_{c_{\alpha \beta}}, v_{c_{\beta \alpha}}$ and $v_{c_{\beta \beta}}$. In the general case, the CCVs depending on $P_{\alpha 0}$ and $P_{\beta 0}$ can lead to large voltage fluctuations when $f_{m}=0$ and the ones depending on $P_{0 \alpha}$ and $P_{0 \beta}$ when $f_{g}=0$. However, for drives applications the machine back emf is very small when $f_{m}=0$ and consequently the power oscillations at this operating point are relatively simple to control. Moreover, for the studied system, the grid frequency is $50 \mathrm{~Hz}$ and the case of $f_{g}=0$ is not applicable.

\section{Vector Model of the $M^{3} C$}

Based on (4), it is concluded that the CCVs are composed of several terms oscillating with different frequencies. Consequently, it is not straightforward to regulate them by using a simple controller. For this reason, an additional linear transformation, denoted $\Sigma \Delta$ transformation, can be applied to enable a vector representation of the Power-CCV model, where the CCVs are represented by expressions with only one fluctuating frequency. The $\Sigma \Delta$ transformation was proposed in [18] to obtain a geometrical orientation of the four circulating currents of the $M^{3} C$. Additionally, a similar transformation has been introduced previously in $M^{2} C$ control systems to consider the interaction of the electrical variables among the converter upper and lower clusters [21], [22]. The $\Sigma \Delta$ transformation is defined as:

$$
\left[\begin{array}{c}
X_{1 \alpha}^{\Sigma \Delta} \\
X_{1 \beta}^{\Sigma \Delta} \\
X_{2 \alpha}^{\Sigma \Delta} \\
X_{2 \beta}^{\Sigma \Delta}
\end{array}\right]=\frac{1}{2}\left[\begin{array}{cccc}
1 & 0 & 0 & 1 \\
0 & 1 & -1 & 0 \\
1 & 0 & 0 & -1 \\
0 & 1 & 1 & 0
\end{array}\right]\left[\begin{array}{c}
X_{\alpha \alpha} \\
X_{\alpha \beta} \\
X_{\beta \alpha} \\
X_{\beta \beta}
\end{array}\right]
$$

The $\Sigma \Delta$ transformation is applied to the terms $v_{c_{\alpha \alpha}}, v_{c_{\alpha \beta}}$, $v_{c_{\beta \alpha}}$ and $v_{c_{\beta \beta}}$. Rewriting (2), and considering these new components yields the Power-CCV model of the $M^{3} C$ in $\Sigma \Delta$ double- $\alpha \beta 0$ coordinates:

$$
C v_{c}^{*} \frac{d}{d t}\left[\begin{array}{ccc}
v_{c_{1 \alpha}}^{\Sigma \Delta} & v_{c_{1 \beta}}^{\Sigma \Delta} & v_{c_{0 \alpha}} \\
v_{c_{2 \alpha}}^{\Sigma \Delta} & v_{c_{2 \beta}}^{\Sigma \Delta} & v_{c_{0 \beta}} \\
v_{c_{\alpha} 0} & v_{c_{\beta 0}} & v_{c_{00}}
\end{array}\right] \approx\left[\begin{array}{ccc}
P_{1 \alpha}^{\Sigma \Delta} & P_{1 \beta}^{\Sigma \Delta} & P_{0 \alpha} \\
P_{2 \alpha}^{\Sigma \Delta} & P_{2 \beta}^{\Sigma \Delta} & P_{0 \beta} \\
P_{\alpha 0} & P_{\beta 0} & P_{00}
\end{array}\right]
$$

The new power-terms on the right-hand side of (6) can also be expressed as a function of the transformed currents and voltages of the converter as follows:

$$
\begin{aligned}
& P_{1 \alpha}^{\Sigma \Delta}=\overbrace{\frac{\left(v_{m \alpha} i_{g \alpha}-v_{g \alpha} i_{m \alpha}\right)+\left(v_{m \beta} i_{g \beta}-v_{g \beta} i_{m \beta}\right)}{6}}^{\mathrm{NC}} \\
& +\overbrace{\frac{\left(v_{m \alpha} i_{2 \alpha}^{\Sigma \Delta}-v_{m \beta} i_{2 \beta}^{\Sigma \Delta}\right)+\left(-v_{g \alpha} i_{2 \alpha}^{\Sigma \Delta}+v_{g \beta} i_{2 \beta}^{\Sigma \Delta}\right)}{\sqrt{6}}}^{\mathrm{SC}}-\overbrace{v_{n} i_{1 \alpha}^{\Sigma \Delta}}^{\mathrm{FC}} \\
& P_{1 \beta}^{\Sigma \Delta}=\frac{\left(v_{m \alpha} i_{g \beta}-v_{g \beta} i_{m \alpha}\right)-\left(v_{m \beta} i_{g \alpha}-v_{g \alpha} i_{m \beta}\right)}{6} \\
& +\frac{\left(v_{m \alpha} i_{2 \beta}^{\Sigma \Delta}+v_{m \beta} i_{2 \alpha}^{\Sigma \Delta}\right)+\left(v_{g \alpha} i_{2 \beta}^{\Sigma \Delta}+v_{g \beta} i_{2 \alpha}^{\Sigma \Delta}\right)}{\sqrt{6}}-v_{n} i_{1 \beta}^{\Sigma \Delta} \\
& P_{2 \alpha}^{\Sigma \Delta}=\frac{\left(v_{m \beta} i_{g \alpha}-v_{g \alpha} i_{m \beta}\right)-\left(v_{m \beta} i_{g \beta}-v_{g \beta} i_{m \beta}\right)}{6} \\
& +\frac{\left(v_{m \alpha} i_{1 \alpha}^{\Sigma \Delta}+v_{m \beta} i_{1 \beta}^{\Sigma \Delta}\right)+\left(-v_{g \alpha} i_{1 \alpha}^{\Sigma \Delta}+v_{g \beta} i_{1 \beta}^{\Sigma \Delta}\right)}{\sqrt{6}}-v_{n} i_{2 \alpha}^{\Sigma \Delta} \\
& P_{2 \beta}^{\Sigma \Delta}=\frac{\left(v_{m \beta} i_{g \beta}-v_{g \beta} i_{m \beta}\right)+\left(v_{m \beta} i_{g \alpha}-v_{g \alpha} i_{m \beta}\right)}{6} \\
& +\frac{\left(v_{m \alpha} i_{1 \beta}^{\Sigma \Delta}-v_{m \beta} i_{1 \alpha}^{\Sigma \Delta}\right)+\left(v_{g \alpha} i_{1 \beta}^{\Sigma \Delta}+v_{g \beta} i_{1 \alpha}^{\Sigma \Delta}\right)}{\sqrt{6}}-v_{n} i_{2 \beta}^{\Sigma \Delta}
\end{aligned}
$$


$P_{\beta 0}=-\frac{\left(v_{m \alpha} i_{m \beta}+v_{m \beta} i_{m \alpha}\right)}{3 \sqrt{2}}-\frac{v_{g \alpha}\left(-i_{1 \beta}^{\Sigma \Delta}+i_{2 \beta}^{\Sigma \Delta}\right)+v_{g \beta}\left(i_{1 \alpha}^{\Sigma \Delta}-i_{2 \alpha}^{\Sigma \Delta}\right)}{\sqrt{3}}-\frac{v_{n} i_{m}}{\sqrt{3}}$
$P_{0 \alpha}=-\frac{\left(v_{g \alpha} i_{g \alpha}-v_{g \beta} i_{g \beta}\right)}{3 \sqrt{2}}+\frac{v_{m \alpha}\left(i_{1 \alpha}^{\Sigma \Delta}+i_{2 \alpha}^{\Sigma \Delta}\right)+v_{m \beta}\left(-i_{1 \beta}^{\Sigma \Delta}+i_{2 \beta}^{\Sigma \Delta}\right)}{\sqrt{3}}-\frac{v_{n} i_{g \alpha}}{\sqrt{3}}$
$P_{0 \beta}=\frac{\left(v_{g \alpha} i_{g \beta}+v_{g \beta} i_{g \alpha}\right)}{3 \sqrt{2}}+\frac{v_{m \alpha}\left(i_{1 \beta}^{\Sigma \Delta}+i_{2 \beta}^{\Sigma \Delta}\right)+v_{m \beta}\left(i_{1 \alpha}^{\Sigma \Delta}-i_{2 \alpha}^{\Sigma \Delta}\right)}{\sqrt{3}}-\frac{v_{n} i_{g \beta}}{\sqrt{3}}$

At this point, the following classifications for the components of the cluster power terms are defined:

- Non-Controllable (NC) Components. These components depend on the machine or grid voltages and currents and cannot be regulated without affecting either the input/output ports.

- Semi-Controllable (SC) Components. These components arise due to interactions between circulating currents or common-mode voltage with the input/output ports currents and voltages.

- Fully-Controllable (FC) Components. These components arise due to interactions between common-mode voltage and circulating currents.

Analysing the power terms, it is possible to identify the unstable operating points. For instance, inserting (7) into (6) and solving for $v_{c_{1 \alpha}}^{\Sigma \Delta}$, the low-frequency component that might lead to large oscillations can be approximated as follows:

$\tilde{v}_{c_{1 \alpha}}^{\Sigma \Delta} \approx \frac{V_{m} I_{g} \sin \left(\phi_{g}+\left(\omega_{g}-\omega_{m}\right) t\right)+V_{g} I_{m} \sin \left(\phi_{m}-\left(\omega_{g}-\omega_{m}\right) t\right)}{6 C v_{c}^{*}\left(\omega_{g}-\omega_{m}\right)}$

From (15), it is concluded that $v_{c_{1 \alpha}}^{\Sigma \Delta}$ has large voltage oscillations when $f_{m} \approx f_{g}$. Extending the previous analysis to the remaining seven $\mathrm{CCV}$ terms (i.e. $v_{c_{1 \beta}}^{\Sigma \Delta}, v_{c_{2 \alpha} \alpha}^{\Sigma \Delta}, v_{c_{2 \beta}}^{\Sigma \Delta}, v_{c_{\alpha 0}}, v_{c_{\beta 0}}$, $\left.v_{c_{0 \alpha}}, v_{c_{0 \beta}}\right)$, the following conclusions are obtained:

- large oscillations are produced in $v_{c_{1 \beta}}^{\Sigma \Delta}$ when $f_{m} \approx f_{g}$.

- large oscillations are produced in $v_{c_{2 \alpha}}^{\Sigma \Delta}$ and $v_{c_{2 \beta}}^{\Sigma \Delta}$ when $f_{m} \approx-f_{g}$.

- large oscillations could be produced in $v_{c_{\alpha 0}}, v_{c_{\beta 0}}$ when $f_{m} \approx 0$.

- large oscillations could be produced in $v_{c_{0 \alpha}}, v_{c_{0 \beta}}$ when $f_{g} \approx 0$.

As mentioned previously the condition when $f_{m} \approx 0$ is not an important issue in drives applications because at this operating point the machine back-emf is very low, and this implies a low value of the power calculated from $V_{m} I_{m}$ [7]. The case of $f_{g} \approx 0$ is not applicable for grid-connected applications.

The use of the $\Sigma \Delta$ transformation enables better representation of the $\mathrm{CCVs}$ regarding the input and output port frequencies because a pair of CCV terms is obtained for each unstable condition. Furthermore, it is possible to represent the dynamics of the $\mathrm{CCV}$ using just four equations. The Vector Power-CCV model of the $M^{3} C$ in $\Sigma \Delta$ double- $\alpha \beta 0$ coordinates is then obtained as:

$$
\begin{aligned}
C v_{c}^{*} \frac{d \boldsymbol{v}_{c_{1 \alpha \beta}}^{\Sigma \Delta} \approx}{d t} \approx & \frac{1}{6}\left(\boldsymbol{v}_{m_{\alpha \beta}}^{c} \boldsymbol{i}_{g_{\alpha \beta}}-\boldsymbol{v}_{g_{\alpha \beta}} \boldsymbol{i}_{m_{\alpha \beta}}^{c}\right) \\
& +\frac{1}{\sqrt{6}}\left(\boldsymbol{v}_{m_{\alpha \beta}} \boldsymbol{i}_{2 \alpha \beta}^{\Sigma \Delta}-\boldsymbol{v}_{g_{\alpha \beta}}^{c} \boldsymbol{i}_{2 \alpha \beta}^{\Sigma \Delta^{c}}\right)-v_{n} \boldsymbol{i}_{1 \alpha \beta}^{\Sigma \Delta} \\
C v_{c}^{*} \frac{d \boldsymbol{v}_{c_{2 \alpha \beta} \Delta}^{\Sigma \Delta} \approx}{d t} & \frac{1}{6}\left(\boldsymbol{v}_{m_{\alpha \beta}} \boldsymbol{i}_{g_{\alpha \beta}}-\boldsymbol{v}_{g_{\alpha \beta}} \boldsymbol{i}_{m_{\alpha \beta}}\right) \\
& +\frac{1}{\sqrt{6}}\left(\boldsymbol{v}_{m_{\alpha \beta}}^{c} \boldsymbol{i}_{1 \alpha \beta}^{\Sigma \Delta}-\boldsymbol{v}_{g_{\alpha \beta}}^{c} \boldsymbol{i}_{1 \alpha \beta}^{\Sigma \Delta^{c}}\right)-v_{n} \boldsymbol{i}_{2 \alpha \beta}^{\Sigma \Delta}
\end{aligned}
$$

$$
\begin{aligned}
C v_{c}^{*} \frac{d \boldsymbol{v}_{c_{0}}^{\alpha \beta} \approx}{d t} \approx & \frac{1}{3 \sqrt{2}}\left(\boldsymbol{v}_{m_{\alpha \beta}}^{c} \boldsymbol{i}_{m_{\alpha \beta}}^{c}\right) \\
& -\frac{1}{\sqrt{3}}\left(\boldsymbol{v}_{g_{\alpha \beta}} \boldsymbol{i}_{1 \alpha \beta}^{\Sigma \Delta^{c}}+\boldsymbol{v}_{g_{\alpha \beta}}^{c} \boldsymbol{i}_{2 \alpha \beta}^{\Sigma \Delta}\right)-\frac{1}{\sqrt{3}} v_{n} \boldsymbol{i}_{m_{\alpha \beta}} \\
C v_{c}^{*} \frac{d \boldsymbol{v}_{c_{\alpha \beta}}^{0} \approx}{d t} \approx & \frac{-1}{3 \sqrt{2}}\left(\boldsymbol{v}_{g_{\alpha \beta}}^{c} \boldsymbol{i}_{g_{\alpha \beta}}^{c}\right) \\
& +\frac{1}{\sqrt{3}}\left(\boldsymbol{v}_{m_{\alpha \beta}} \boldsymbol{i}_{1 \alpha \beta}^{\Sigma \Delta}+\boldsymbol{v}_{m_{\alpha \beta}}^{c} \boldsymbol{i}_{2 \alpha \beta}^{\Sigma \Delta}\right)-\frac{1}{\sqrt{3}} v_{n} \boldsymbol{i}_{g_{\alpha \beta}}
\end{aligned}
$$

where the superscript $c$ represents the complex conjugate operator and (16)-(19) represent the vector Power-CCV model of the $M^{3} C$. Additionally, vector notation is used as follows:

$$
\begin{aligned}
\boldsymbol{P}_{1 \alpha \beta}^{\Sigma \Delta} & =P_{1 \alpha}^{\Sigma \Delta}+j P_{1 \beta}^{\Sigma \Delta} ; \boldsymbol{P}_{2 \alpha \beta}^{\Sigma \Delta}=P_{2 \alpha}^{\Sigma \Delta}+j P_{2 \beta}^{\Sigma \Delta} \\
\boldsymbol{P}_{0}^{\alpha \beta} & =P_{\alpha 0}+j P_{\beta 0} ; \boldsymbol{P}_{\alpha \beta}^{0}=P_{0 \alpha}+j P_{0 \beta} \\
\boldsymbol{v}_{c_{1 \alpha \beta}}^{\Sigma \Delta} & =v_{c_{1 \alpha}}^{\Sigma \Delta}+j v_{c_{1 \beta}}^{\Sigma \Delta} ; \boldsymbol{v}_{c_{2 \alpha \beta}}^{\Sigma \Delta}=v_{c_{2 \alpha}}^{\Sigma \Delta}+j v_{c_{2 \beta}}^{\Sigma \Delta} \\
\boldsymbol{v}_{m_{\alpha \beta}} & =v_{m \alpha}+j v_{m \beta} ; \boldsymbol{i}_{m_{\alpha \beta}}=i_{m \alpha}+j i_{m \beta} \\
\boldsymbol{v}_{g_{\alpha \beta}} & =v_{g \alpha}+j v_{g \beta} ; \boldsymbol{i}_{g_{\alpha \beta}}=i_{g \alpha}+j i_{g \beta}
\end{aligned}
$$

where $\boldsymbol{v}_{m_{\alpha \beta}}$ and $\boldsymbol{i}_{m_{\alpha \beta}}$ represent the input-port voltages and currents in $\alpha \beta 0$ frame. Similarly, $\boldsymbol{v}_{g_{\alpha \beta}}$ and $\boldsymbol{i}_{g_{\alpha \beta}}$ represent the output-port voltages and currents.

The vector $\boldsymbol{v}_{c_{1 \alpha \beta}}^{\Sigma \Delta}$ has a frequency of $f_{m}-f_{g}, \boldsymbol{v}_{c_{2 \alpha \beta}}^{\Sigma \Delta}$ has a frequency of $f_{m}+f_{g}, \boldsymbol{v}_{c_{0}}^{\alpha \beta}$ has a frequency of $2 f_{m}$, and $\boldsymbol{v}_{c_{\alpha \beta}}^{0}$ has a frequency of $2 f_{g}$. This four vector equations allow a simple analysis and implementation of Vector Control structures to regulate the floating capacitor voltages as discussed in the next Section. The previous classification (i.e. NC, SC and FC) is valid for the CCV vectors as well.

\section{Vector Control Systems of the $M^{3} C$}

The proposed control system comprises a nested structure for decoupled regulation of the CCVs and for the input-output ports variables. Each controller is described in the following subsections.

\section{A. Vector Control System of the CCVs}

A Vector Control System for the regulation of $\boldsymbol{v}_{c_{1 \alpha \beta} \Delta}^{\Sigma \Delta}$, $\boldsymbol{v}_{c_{2 \alpha \beta}}^{\Sigma \Delta}, \boldsymbol{v}_{c_{0}}^{\alpha \beta}, \boldsymbol{v}_{c_{\alpha \beta}}^{0}$ is proposed. The average value of all the floating capacitor voltages is regulated using the component $v_{c_{00}}$. For the other voltage components in the $\Sigma \Delta$ double$\alpha \beta 0$ coordinates, the control strategy applied depends on the operating mode. In DFM operation, it is assumed that the capacitor value is sufficient to attenuate the voltage oscillations produced at $\left(2 f_{g}, 2 f_{m}, f_{g} \pm f_{m}\right)$ and only the average values of the voltages in the $\Sigma \Delta$ double- $\alpha \beta 0$ coordinates have to be regulated to zero using the SC terms of (16)-(19). In EFM, as well as regulating the average values, the low frequency oscillations of frequency $f_{g} \pm f_{m}$ are regulated to zero using the FC terms of (16)-(19). The frequency $f_{m}^{\max }$ is defined as the maximum frequency at which to switch between DFM and EFM. The selection of $f_{m}^{\max }$ depends on the parameters of the converter such as cell capacitance, input/output port voltages and power factors. An analysis of the influence of these parameters is given in [23]. For this work, $f_{m}^{\max } \approx 0.9 f_{g}$ is used. 


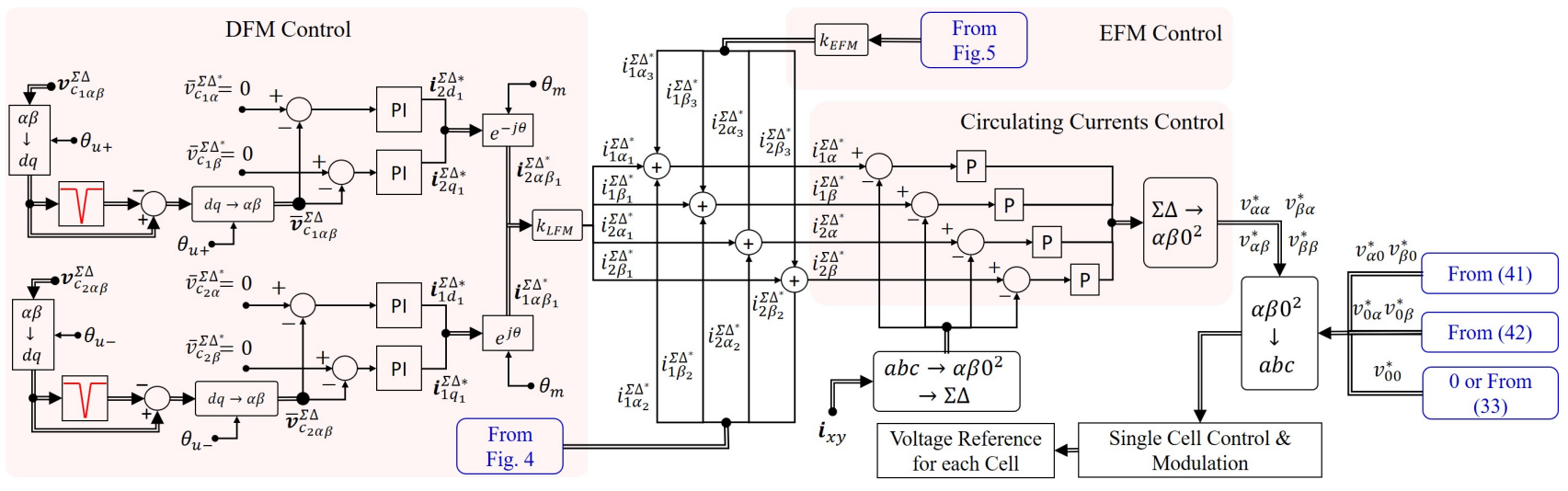

Fig. 3. Proposed Vector Control System for the regulation of the CCVs.

1) Control of the average component of the capacitor voltages

The term $v_{c_{00}}$ represents the average voltage taken across all the floating capacitors of the $M^{3} C$ and it is related to the active power flowing into the converter $P_{00}$. Therefore, the following expression is written:

$$
C v_{c}^{*} \frac{d v_{c_{00}}}{d t} \approx P_{00}=\overbrace{\frac{\left(v_{m \alpha} i_{m \alpha}+v_{m \beta} i_{m \beta}\right)}{3}}^{\text {Input Power }=P_{\text {in }}}-\overbrace{\frac{\left(v_{g \alpha} i_{g \alpha}+v_{g \beta} i_{g \beta}\right)}{3}}^{\text {Output Power }=P_{\text {out }}}
$$

The term $P_{\text {out }}$ represents the $M^{3} C$ output power, and it is considered as a disturbance that can be fed-forward. Additionally, the input-port variables can be referred to a $d q$ frame rotating at $\theta_{m}$ and oriented along the voltage vector $\boldsymbol{v}_{m}$. Therefore, (21) becomes:

$$
C v_{c}^{*} \frac{d v_{c_{00}}}{d t} \approx P_{00}=\frac{1}{3} v_{m d} i_{m d 1}
$$

The total input-port current reference discussed in Section IV.C [see (41)] is then composed of $i_{m d 1}$ plus the corresponding power producing current related to the power been transferred between both ports. Then, $i_{m d 1}$ is manipulated to regulate the average value of the CCVs.

2) DFM Control of $\boldsymbol{v}_{c_{1 \alpha \beta}}^{\Sigma \Delta}$ and $\boldsymbol{v}_{c_{2 \alpha \beta}}^{\Sigma \Delta}$

As mentioned before, in DFM only the average values of $\boldsymbol{v}_{c_{1 \alpha \beta}}^{\Sigma \Delta}$ and $\boldsymbol{v}_{c_{2 \alpha \beta}}^{\Sigma \Delta}$ are regulated. By using complex polar notation, (16) and (17) can be rewritten as:

$$
\begin{aligned}
C v_{c}^{*} \frac{d \boldsymbol{v}_{c_{1 \alpha \beta}}^{\Sigma \Delta}}{d t} \approx & \frac{1}{6}\left(\boldsymbol{v}_{m_{d q}}^{c} \boldsymbol{i}_{g_{d q}}-\boldsymbol{v}_{g_{d q}} \boldsymbol{i}_{m_{d q}}^{c}\right) \mathrm{e}^{j\left(\theta_{g}-\theta_{m}\right)} \\
& +\frac{1}{\sqrt{6}}\left(\boldsymbol{v}_{m_{d q}} \boldsymbol{i}_{2 d q}^{\Sigma \Delta}-\boldsymbol{v}_{g_{d q}} \boldsymbol{i}_{2 d q}^{\Sigma \Delta c} \mathrm{e}^{-j\left(\theta_{g}-\theta_{m}\right)}\right) \\
C v_{c}^{*} \frac{d \boldsymbol{v}_{c_{2 \alpha \beta}}^{\Sigma \Delta}}{d t} \approx & \frac{1}{6}\left(\boldsymbol{v}_{m_{d q}} \boldsymbol{i}_{g_{d q}}-\boldsymbol{v}_{g_{d q}} \boldsymbol{i}_{m_{d q}}\right) \mathrm{e}^{j\left(\theta_{g}+\theta_{m}\right)} \\
& +\frac{1}{\sqrt{6}}\left(\boldsymbol{v}_{m_{d q}}^{c} \boldsymbol{i}_{1 d q}^{\Sigma \Delta}-\boldsymbol{v}_{g_{d q}}^{c} \boldsymbol{i}_{1 d q}^{\Sigma \Delta c} \mathrm{e}^{j\left(-\theta_{g}+\theta_{m}\right)}\right)
\end{aligned}
$$

where each vector in $\Sigma \Delta$ double- $\alpha \beta 0$ coordinates has been defined as $\boldsymbol{x}_{\alpha \beta}^{\Sigma \Delta}=\boldsymbol{x}_{d q}^{\Sigma \Delta} e^{j \theta_{e}}$, and $\theta_{e}$ is the angle of the vector $\boldsymbol{x}$, and the common-mode voltage is zero.

The circulating currents are defined as follows to produce dc components in (23)-(24):

$$
\boldsymbol{i}_{1 \alpha \beta_{1}}^{\Sigma \Delta *}=\boldsymbol{i}_{1 d q_{1}}^{\Sigma \Delta *} \mathrm{e}^{j \theta_{m}} ; \boldsymbol{i}_{2 \alpha \beta_{1}}^{\Sigma \Delta *}=\boldsymbol{i}_{2 d q_{1}}^{\Sigma \Delta *} \mathrm{e}^{-j \theta_{m}}
$$

Where $\boldsymbol{i}_{1 \alpha \beta_{1}}^{\Sigma \Delta *}$ and $\boldsymbol{i}_{2 \alpha \beta_{1}}^{\Sigma \Delta *}$ represent components of the total circulating current references. Inserting (25) into (23)-(24) and filtering the ac components yields:

$$
C v_{c}^{*} \frac{d}{d t} \overline{\boldsymbol{v}}_{c_{1 \alpha \beta} \Delta}^{\Sigma \Delta} \approx \frac{\boldsymbol{v}_{m_{d q}} \boldsymbol{i}_{2 d q_{1}}^{\Sigma \Delta *}}{\sqrt{6}} ; C v_{c}^{*} \frac{d}{d t} \overline{\boldsymbol{v}}_{c_{2 \alpha \beta} \Delta}^{\Sigma \Delta} \approx \frac{\boldsymbol{v}_{m_{d q}}^{c} \boldsymbol{i}_{1 d q_{1}}^{\Sigma \Delta *}}{\sqrt{6}}
$$

$\overline{\boldsymbol{v}}_{c_{1 \alpha \beta}}^{\Sigma \Delta}$ and $\overline{\boldsymbol{v}}_{c_{2 \alpha \beta}}^{\Sigma \Delta}$ are the filtered versions of $\boldsymbol{v}_{c_{1 \alpha \beta}}^{\Sigma \Delta}$ and $\boldsymbol{v}_{c_{2 \alpha \beta}}^{\Sigma \Delta}$.

The active power flows produced by the right-side components of (26) are used to regulate the average component of $\boldsymbol{v}_{c_{1 \alpha \beta}}^{\Sigma \Delta}$ and $\boldsymbol{v}_{c_{2 \alpha \beta}}^{\Sigma \Delta}$. Notice that in (26) to maximise the power regulation effect produced by the circulating current, the currents $i_{1 \alpha \beta_{1}}^{\Sigma \Delta *}$ and $i_{2 \alpha \beta_{1}}^{\Sigma \Delta *}$ are controlled to have zero phase shift with respect to the input-port voltages.

Fig. 3 shows the proposed balancing control system. The average components of both vectors are obtained using synchronous reference frame notch filters [3]. Accordingly, $\boldsymbol{v}_{c_{1 \alpha \beta} \Delta}^{\Sigma \Delta}$ is referred to a $d q$-frame rotating at $\theta_{u^{+}}=\int\left(\omega_{g}-\omega_{m}\right) d t$, and $\boldsymbol{v}_{c_{2 \alpha \beta}}^{\Sigma \Delta}$ is referred to a $d q$-frame rotating at $\theta_{u-}=\int\left(\omega_{g}+\omega_{m}\right) d t$. The oscillatory components are filtered and both components are rotated back to obtain $\overline{\boldsymbol{v}}_{c_{1 \alpha \beta}}^{\Sigma \Delta}$ and $\overline{\boldsymbol{v}}_{c_{2 \alpha \beta}}^{\Sigma \Delta}$. Then, outer control loops regulate $\overline{\boldsymbol{v}}_{c_{1 \alpha \beta}}^{\Sigma \Delta}$ and $\overline{\boldsymbol{v}}_{c_{2 \alpha \beta} \Delta}^{\Sigma \Delta}$ to zero using PI controllers. The outputs of these controllers are used to calculate the reference of the $d q$ circulating currents in accordance with (25). Additionally, the gains to enable the DFM control are $k_{D F M}=1$ and $k_{E F M}=0$.

3) DFM Control of $\boldsymbol{v}_{c_{0}}^{\alpha \beta}$ and $\boldsymbol{v}_{c_{\alpha \beta}}^{0}$

Similar assumptions to those used in Sub-Section IV-A2 are valid for the regulation of (18)-(19). Vectors $\boldsymbol{v}_{c_{0}}^{\alpha \beta}$ and $\boldsymbol{v}_{c_{\alpha \beta}}^{0}$ are rewritten as follows:

$$
\begin{gathered}
C v_{c}^{*} \frac{d \boldsymbol{v}_{c_{0}}^{\alpha \beta}}{d t} \approx \frac{\left(\boldsymbol{v}_{m_{d q}}^{c} \boldsymbol{i}_{m_{d q}}^{c}\right) e^{-j 2 \theta_{m}}}{3 \sqrt{2}}-\frac{\left(\boldsymbol{v}_{g_{d q}} \boldsymbol{i}_{1 d q}^{\Sigma \Delta c}+\boldsymbol{v}_{g_{d q}}^{c} \boldsymbol{i}_{2 d q}^{\Sigma \Delta}\right)}{\sqrt{3}} \\
C v_{c}^{*} \frac{d \boldsymbol{v}_{c_{\alpha \beta}}^{0}}{d t} \approx \frac{-\left(\boldsymbol{v}_{g_{d q}}^{c} \boldsymbol{i}_{g_{d q}}^{c}\right) e^{-j 2 \theta_{g}}}{3 \sqrt{2}}+\frac{\left(\boldsymbol{v}_{m_{d q}} \boldsymbol{i}_{1 d q}^{\Sigma \Delta}+\boldsymbol{v}_{m_{d q}}^{c} \boldsymbol{i}_{2 d q}^{\Sigma \Delta}\right)}{\sqrt{3}}
\end{gathered}
$$

The circulating currents are defined as follows to produce $\mathrm{dc}$ components in (27)-(28):

$$
\boldsymbol{i}_{1 \alpha \beta_{2}}^{\Sigma \Delta *}=\boldsymbol{i}_{1 d q_{2}}^{\Sigma \Delta *} \mathrm{e}^{j \theta_{g}} ; \boldsymbol{i}_{2 \alpha \beta_{2}}^{\Sigma \Delta *}=\boldsymbol{i}_{2 d q_{2}}^{\Sigma \Delta *} \mathrm{e}^{j \theta_{m}}
$$

Where $i_{1 \alpha \beta_{2}}^{\Sigma \Delta *}$ and $i_{2 \alpha \beta_{2}}^{\Sigma \Delta *}$ represent components of the total circulating current references.

Substituting (29) in (27)-(28) and filtering the ac components yields: 


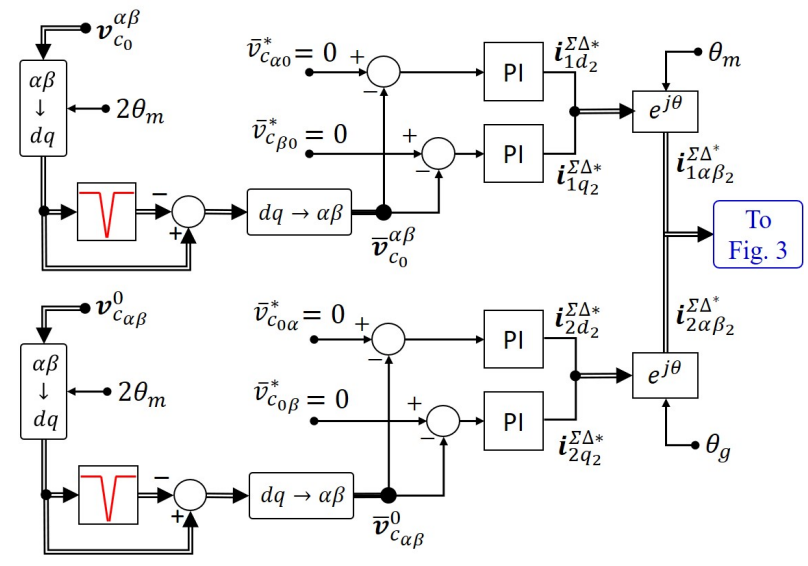

Fig. 4. Proposed DFM Control of $\boldsymbol{v}_{c_{0}}^{\alpha \beta}$ and $\boldsymbol{v}_{c_{\alpha \beta}}^{0}$.

$$
C v_{c}^{*} \frac{d}{d t} \overline{\boldsymbol{v}}_{c_{0}}^{\alpha \beta} \approx-\frac{\boldsymbol{v}_{g_{d q}} \boldsymbol{i}_{1 d q_{2}}^{\Sigma \Delta c}}{\sqrt{3}} ; C v_{c}^{*} \frac{d}{d t} \overline{\boldsymbol{v}}_{c_{\alpha \beta}}^{0} \approx-\frac{\boldsymbol{v}_{m_{d q}}^{c} \boldsymbol{i}_{2 d q_{2}}^{\Sigma \Delta}}{\sqrt{3}}
$$

Again it is assumed that the active power flows are produced in the right-hand side terms of (30) by currents and voltages which are in phase. The proposed DFM control system is presented in Fig. 4 (it has the same structure as the control system depicted in Fig. 3). The average components $\overline{\boldsymbol{v}}_{c_{0}}^{\alpha \beta}$ are regulated to zero using circulating currents at $\omega_{g}$, whereas the average components $\overline{\boldsymbol{v}}_{c_{\alpha \beta}}^{0}$ are regulated to zero using circulating currents at $\omega_{m}$.

\section{B. Open-Loop EFM Control of $\boldsymbol{v}_{c_{1 \alpha \beta}}^{\Sigma \Delta}$ and $\boldsymbol{v}_{c_{2 \alpha \beta}}^{\Sigma \Delta}$}

In EFM, the CCV oscillations can be partially mitigated by imposing operational restrictions on the input/output ports [14], [17]. Moreover there are other methods that use only circulating currents [15], and a combination of circulating currents and common-mode voltage to mitigate the voltage oscillations [17], [18].

In previous works [14], [17] the values of $Q_{m}=-Q_{g}$ and $V_{m}=V_{g}$ are selected for equal frequency operation, in addition to the regulation of the average component of the $\mathrm{CCV}$ vectors. Note that in these cases, mitigation signals are applied (during EFM) in an open-loop manner, because large instantaneous voltage oscillations are not directly controlled to manipulate the values of $Q_{g}$ and $V_{m}$. Therefore, this is an intrinsically open-loop control method which cannot compensate changes in the operating points, incorrect estimation of the reactive power, measurement errors, etc.

\section{Closed-loop EFM Control of $\boldsymbol{v}_{c_{1 \alpha \beta}}^{\Sigma \Delta}$ and $\boldsymbol{v}_{c_{2 \alpha \beta}}^{\Sigma \Delta}$}

The open-loop EFM control is not always practicable and any drift or difference in $Q_{m}=-Q_{g}$ and/or $V_{m}=V_{g}$ could produce significant low-frequency capacitor voltage oscillations [17]. Therefore, to ensure good performance of the $M^{3} C$ under different operational conditions, a new closedloop control system is proposed to operate in the EFM without operational restrictions. Accordingly, the control flexibility is increased and the $M^{3} C$ can operate in EFM with different power factors and voltage amplitudes. In this proposal, the $\mathrm{CCV}$ vectors are regulated using closed-loop vector-control algorithms implemented in synchronously rotating $d q$ frames.
As the proposed control system is implemented in $d q$ coordinates, (16) is referred to a $d q$-frame using $\theta_{u+}=\int\left(\omega_{g}-\omega_{m}\right) d t$, and (17) is referred to a $d q$-frame rotating using $\theta_{u^{-}}=\int\left(\omega_{g}+\omega_{m}\right) d t$.

$$
\begin{gathered}
C v_{c}^{*}\left(\frac{d \boldsymbol{v}_{c_{1 d q}}^{\Sigma \Delta}}{d t}+j \omega_{u \Upsilon} \boldsymbol{v}_{c_{1 d q}}^{\Sigma \Delta}\right) \approx \frac{1}{6}\left(\boldsymbol{v}_{m_{d q}}^{c} \boldsymbol{i}_{g_{d q}}-\boldsymbol{v}_{g_{d q}} \boldsymbol{i}_{m_{d q}}^{c}\right) \\
+\frac{1}{\sqrt{6}}\left(\boldsymbol{v}_{m_{d q}} \boldsymbol{i}_{2_{d q}}^{\Sigma \Delta} e^{j 3 \theta_{m}}-\boldsymbol{v}_{g_{d q}}^{c} \boldsymbol{i}_{2_{d q}}^{\Sigma \Delta c} e^{-j 3 \theta_{g}}\right)-v_{n} \boldsymbol{i}_{1_{d q}}^{\Sigma \Delta} \\
C v_{c}^{*}\left(\frac{d \boldsymbol{v}_{c_{2 d q}}^{\Sigma \Delta}}{d t}+j \omega_{u-} \boldsymbol{v}_{c_{2 d q}}^{\Sigma \Delta}\right) \approx \frac{1}{6}\left(\boldsymbol{v}_{m_{d q}} \boldsymbol{i}_{g_{d q}}-\boldsymbol{v}_{g_{d q}} \boldsymbol{i}_{m_{d q}}\right) \\
+\frac{1}{\sqrt{6}}\left(\boldsymbol{v}_{m_{d q}}^{c} \boldsymbol{i}_{1_{d q} \Delta \Delta}^{\Sigma \Delta} e^{-j 3 \theta_{m}}-\boldsymbol{v}_{g_{d q}}^{c} \boldsymbol{i}_{1_{d q}}^{\Sigma \Delta c} e^{-j 3 \theta_{g}}\right)-v_{n} \boldsymbol{i}_{2_{d q}}^{\Sigma \Delta}
\end{gathered}
$$

Again the common-mode voltage and the $d q$ circulating currents should be in phase to efficiently produce adjustable power flows. Additionally, $i_{1_{d q} \Delta}^{\Sigma \Delta}$ and $i_{2_{d q} \Delta}^{\Sigma \Delta}$ have to be defined as relatively high-frequency signals to avoid undesired lowfrequency power flows that could lead to large voltage oscillations. Owing to these considerations, the $d q$ circulating currents and the common-mode voltage are defined as:

$$
\boldsymbol{i}_{1 d q_{3}}^{\Sigma \Delta *}=\boldsymbol{I}_{1 d q_{3}}^{\Sigma{ }^{\Sigma} \Delta *} f(t) ; \boldsymbol{i}_{2 d q_{3}}^{\Sigma \Delta *}=\boldsymbol{I}_{2 d q_{3}}^{\Sigma \Delta *} f(t) ; v_{n}=V_{0} g(t)
$$

Where $v_{n}$ is a square (or trapezoidal) waveform of frequency $\omega_{n}$. The terms $i_{1 d q_{3}}^{\Sigma \Delta *}$ and $\boldsymbol{i}_{2 d q_{3}}^{\Sigma \Delta *}$ represent components of the total circulating current references. The functions $f(t)$ and $g(t)$ are in phase, $f(t)=A_{1} \sin \theta_{n}+A_{3} \sin 3 \theta_{n}$ (where $\theta_{n}=\omega_{n} t$, with $\omega_{n}$ set at a relatively high frequency), and $g(t)=\operatorname{sign}\{f(t)\}$. The amplitudes of the constants $A_{1}, A_{3}$ and $V_{0}$ are chosen to reduce the peak of the circulating currents as proposed in [24]. These definitions imply than the product $f(t) g(t)$ has a large dc component.

Neglecting the high frequency components in (31)-(32):

$$
\begin{gathered}
C v_{c}^{*} \frac{d \boldsymbol{v}_{c_{1 d q}}^{\Sigma \Delta}}{d t} \approx \frac{1}{6}\left(\boldsymbol{v}_{m_{d q}}^{c} \boldsymbol{i}_{g_{d q}}-\boldsymbol{v}_{g_{d q}} \boldsymbol{i}_{m_{d q}}^{c}\right)-V_{0} \boldsymbol{I}_{1 d q_{3}}^{\Sigma \Delta} \\
C v_{c}^{*} \frac{d \boldsymbol{v}_{c_{2 d q}}^{\Sigma \Delta}}{d t} \approx \frac{1}{6}\left(\boldsymbol{v}_{m_{d q}} \boldsymbol{i}_{g_{d q}}-\boldsymbol{v}_{g_{d q}} \boldsymbol{i}_{m_{d q}}\right)-V_{0} \boldsymbol{I}_{2 d q_{3}}^{\Sigma \Delta}
\end{gathered}
$$

To improve the dynamic response, the $\mathrm{NC}$ terms of (34)-(35) can be fed-forward to the total circulating current (see Fig. 5). Therefore:

$$
\begin{gathered}
\boldsymbol{I}_{1 d q_{3}}^{\Sigma \Delta}=\boldsymbol{I}_{1 d q_{3 c}}^{\Sigma \Delta}+\boldsymbol{I}_{1 d q_{3 f}}^{\Sigma \Delta} ; \boldsymbol{I}_{1 d q_{3 f}}^{\Sigma \Delta}=\frac{\left(\boldsymbol{v}_{m_{d q}}^{c} \boldsymbol{i}_{g_{d q}}-\boldsymbol{v}_{g_{d q}} \boldsymbol{i}_{m_{d q}}^{c}\right)}{6 V_{0}} \\
\boldsymbol{I}_{2 d q_{3}}^{\Sigma \Delta}=\boldsymbol{I}_{2 d q_{3 c}}^{\Sigma \Delta}+\boldsymbol{I}_{2 d q_{3 f}}^{\Sigma \Delta} ; \boldsymbol{I}_{2 d q_{3 f}}^{\Sigma \Delta}=\frac{\left(\boldsymbol{v}_{m_{d q}} \boldsymbol{i}_{g_{d q}}-\boldsymbol{v}_{g_{d q}} \boldsymbol{i}_{m_{d q}}\right)}{6 V_{0}}
\end{gathered}
$$

Finally, inserting (36)-(37) into (34)-(35) gives:

$$
C v_{c}^{*} \frac{d \boldsymbol{v}_{c_{1 d q} \Delta}^{\Sigma \Delta}}{d t} \approx-V_{0} \boldsymbol{I}_{1 d q_{3 c}}^{\Sigma \Delta *} ; C v_{c}^{*} \frac{d \boldsymbol{v}_{c_{2 d q}}^{\Sigma \Delta}}{d t} \approx-V_{0} \boldsymbol{I}_{2 d q_{3 c}}^{\Sigma \Delta *}
$$

The control system for EFM operation is presented in Fig. 5. When $f_{m}>f_{m}^{\max }$, the circulating currents of (38) are used to regulate $\boldsymbol{v}_{c_{1 \alpha \beta}}^{\Sigma \Delta}$ and $\boldsymbol{v}_{c_{2 \alpha \beta}}^{\Sigma \Delta}$ in the $d q$ frames using PI controllers. The dynamic regulation is improved through feed-forward of the component $\boldsymbol{I}_{1 d q_{3 f}}^{\Sigma \Delta}$, which represents the oscillations of the NC component. The output of the external control loop is multiplied by $f(t)$ and the common-mode voltage is imposed as in (33). In this case, the weighting factors are selected to $k_{D F M}=0$ and $k_{E F M}=1$.

The control schemes used to regulate the voltage oscillations during EFM are shown in Fig. 6. The upper graphic shows 


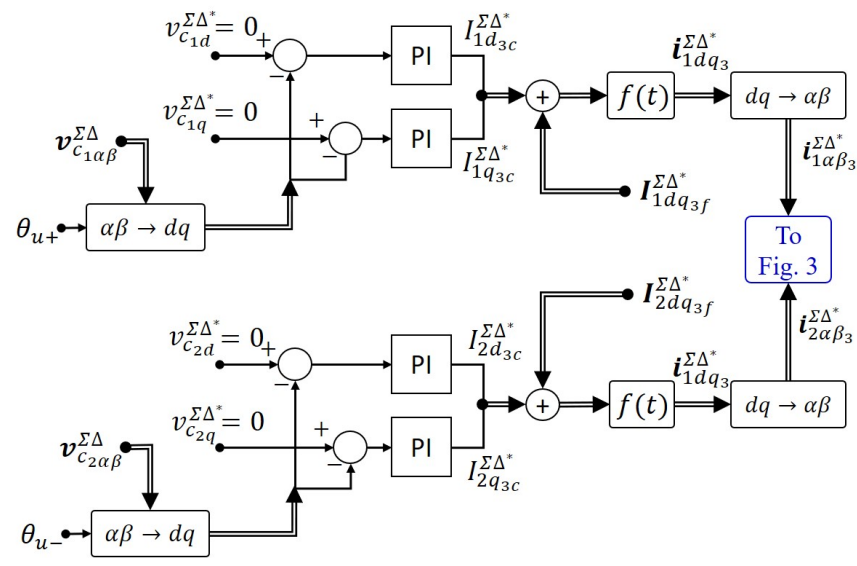

Fig. 5. Proposed EFM Control of $\boldsymbol{v}_{c_{1 \alpha \beta}}^{\Sigma \Delta}$ and $\boldsymbol{v}_{c_{2 \alpha \beta}}^{\Sigma \Delta}$.

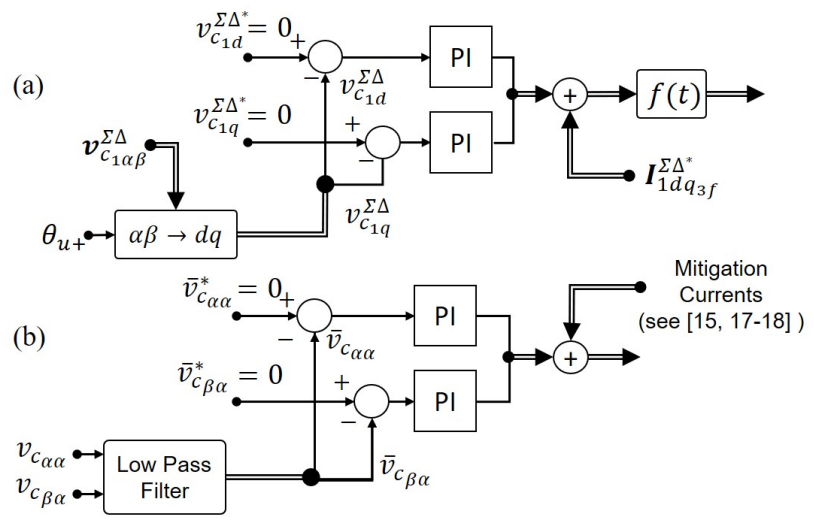

Fig. 6. (a) Proposed closed-loop EFM control system. (b) Open-Loop EFM Control Strategies.

the control system proposed in this work where the voltage oscillations in $\boldsymbol{v}_{c_{1 \alpha \beta}}^{\Sigma \Delta}$ are directly regulated using closed loop control. The lower graphic in Fig. 6 shows the control scheme reported in the literature, where mitigation signals are offline calculated and applied in an open-loop fashion.

\section{Circulating Current Control}

In Fig. 3 the overall control system is shown. The cluster currents are transformed to $\Sigma \Delta$ double- $\alpha \beta 0$ coordinates to be compared to the total circulating current references which are obtained considering superposition of the references from the CCV vector control systems. For instance, $i_{1 \alpha \beta}^{\Sigma \Delta *}=i_{1 \alpha \beta_{1}}^{\Sigma \Delta *}+\boldsymbol{i}_{1 \alpha \beta_{2}}^{\Sigma \Delta *}+\boldsymbol{i}_{1 \alpha \beta_{3}}^{\Sigma \Delta *}$. Because of simplicity, proportional controllers are used to track the circulating current references. The output of the proportional controllers are rotated back to Double- $\alpha \beta 0$ coordinates resulting in $v_{\alpha \alpha}^{*}$, $v_{\alpha \beta}^{*}, v_{\beta \alpha}^{*}$, and $v_{\beta \beta}^{*}$. Thereafter, these voltage references are referred to the natural $(a b c-r s t)$ frame to be processed by the single-cell modulation and control stage (see [10]).

\section{E. Input and Output Control Systems}

Using (1), and considering that the voltages connected to the input-output ports are balanced (i.e. $v_{g 0}=v_{m 0}=0$ ) and that there is no current circulation path between the neutral points $N$ and $n$ (i.e. $i_{00}=0$ ), the Voltage-Current model of (1) can be re-written as two independent equations to describe the dynamics of the input and output ports.

The dynamics of the input-port Voltage-Current Model are represented in $d q$ coordinates rotating at $\theta_{m}$ :

$$
\left[\begin{array}{c}
v_{m d} \\
v_{m q}
\end{array}\right]=\frac{1}{\sqrt{3}}\left[\begin{array}{l}
v_{d 0} \\
v_{q 0}
\end{array}\right]+\frac{L_{c}}{3}\left[\begin{array}{cc}
\frac{d}{d t} & -\omega_{m} \\
\omega_{m} & \frac{d}{d t}
\end{array}\right]\left[\begin{array}{c}
i_{m d} \\
i_{m q}
\end{array}\right]
$$

Similarly, the dynamics of the output-port Voltage-Current Model are represented in $d q$ coordinates rotating at $\theta_{g}$ :

$$
-\left[\begin{array}{c}
v_{g d} \\
v_{g q}
\end{array}\right]=\frac{1}{\sqrt{3}}\left[\begin{array}{c}
v_{0 d} \\
v_{0 q}
\end{array}\right]+\frac{L_{c}}{3}\left[\begin{array}{cc}
\frac{d}{d t} & -\omega_{g} \\
\omega_{g} & \frac{d}{d t}
\end{array}\right]\left[\begin{array}{c}
i_{g d} \\
i_{g q}
\end{array}\right]
$$

Then, the input-port and the output-port can be controlled using conventional $d q$ control systems. To achieve decoupled regulation, the voltage commands are:

$$
\begin{gathered}
\frac{1}{\sqrt{3}}\left[\begin{array}{c}
v_{d 0}^{*} \\
v_{q 0}^{*}
\end{array}\right]=\left[\begin{array}{l}
v_{m d} \\
v_{m q}
\end{array}\right]-\left[\begin{array}{cc}
0 & \frac{-L_{c} \omega_{m}}{3} \\
\frac{L_{c} \omega_{m}}{3} & 0
\end{array}\right]\left[\begin{array}{c}
i_{m d} \\
i_{m q}
\end{array}\right]-P I_{m}\left[\begin{array}{c}
i_{m d}^{*}-i_{m d} \\
i_{m q}^{*}-i_{m q}
\end{array}\right] \\
\frac{-1}{\sqrt{3}}\left[\begin{array}{l}
v_{0 d}^{*} \\
v_{0 q}^{*}
\end{array}\right]=\left[\begin{array}{l}
v_{g d} \\
v_{g q}
\end{array}\right]-\left[\begin{array}{cc}
0 & -\frac{L_{c} \omega_{g}}{3} \\
\frac{L_{c} \omega_{g}}{3} & 0
\end{array}\right]\left[\begin{array}{l}
i_{g d} \\
i_{g q}
\end{array}\right]-P I_{g}\left[\begin{array}{c}
i_{g d}^{*}-i_{g d} \\
i_{g q}^{*}-i_{g q}
\end{array}\right]
\end{gathered}
$$

where $P I_{m}$ and $P I_{g}$ stand for the transfer functions of the PI controllers used to regulate the currents at each port. The voltage references obtained in (41)-(42) are rotated back to double $\alpha \beta 0$ frame resulting in $v_{\alpha 0}^{*}-v_{\beta 0}^{*}$ for the input-port, and $v_{0 \alpha}^{*}-v_{0 \beta}^{*}$ for the output-port. These references are sent to the single-cell control stage, as shown in Fig. 3. Note that $i_{m d}^{*}=i_{m d 1}^{*}+i_{m d 2}^{*}$, where $i_{m d 1}^{*}$ is the current used to regulate the average value of the CCVs, and $i_{m d 2}^{*}$ is the power producing current. The output-port current references are calculated to regulate the power transferred from the input to the output port. Different calculations of the current references $i_{m d}^{*}, i_{m q}^{*}, i_{g d}^{*}$ and $i_{q}^{*}$ can be imposed to fulfil the requirements of the application. For example, the input-port control can be as stated in [10] for a wind energy conversion system, and as stated in [5] for an induction machine drive.

\section{F. Single-Cell Control and Modulation Stage}

The voltage references obtained in the control systems above (i.e. $v_{\alpha \alpha}^{*}, v_{\beta \alpha}^{*}, v_{\alpha \beta}^{*}, v_{\beta \beta}^{*}, v_{\alpha 0}^{*}, v_{\beta 0}^{*}, v_{0 \alpha}^{*}$ and $v_{0 \beta}^{*}$ ) are transformed to the natural reference frame using the inverse Double- $\alpha \beta 0$ Transformation. Then, single-cell control proposed in [25] is used. The capacitor voltage of the $i^{t h}$ $[i \in(1, n)]$ cell is compared to the algebraic-mean value of the corresponding CCV. The resulting error is multiplied by the sign of the cluster current to generate either an in-phase or a 180 degrees out-of-phase voltage with respect to the cluster current. Therefore capacitor voltage balancing of that particular cell is produced by supplying or releasing energy to/from the capacitor, driving its voltage to $v_{c}^{*}$. Finally, phaseshifted unipolar sinusoidal PWM is used to generate the switching signals of each cell [25].

\section{Simulation Results}

A $10 \mathrm{MW} M^{3} C$ simulation model has been implemented in PLECS software to validate the theoretical work. The parameters considered are shown in Table I. The control strategies published in [17] were implemented using the power terms produced by the circulating current and the commonmode voltage, and the corresponding results are presented 
TABLE I

Simulation ANd EXPERIMENTAL PROTOTYPE PARAMETERS.

\begin{tabular}{c||c||c} 
Parameters & Simulation & Experimental setup \\
\hline \hline Nominal Power & $10 \mathrm{MVA}$ & $5 \mathrm{kVA}$ \\
\hline Cells per branch & 7 & 3 \\
\hline Input Voltage/Freq. & $5.39 \mathrm{kV} / 40-50 \mathrm{~Hz}$ & $200 \mathrm{~V} /-48$ to $50 \mathrm{~Hz}$ \\
\hline Cluster Inductor & $1.3 \mathrm{mH}$ & $2.5 \mathrm{mH}$ \\
\hline Single cell $C$. & $7 \mathrm{mF}$ & $4.7 \mathrm{mF}$ \\
\hline Capacitor Voltage & $2 \mathrm{kV}$ & $150 \mathrm{~V}$ \\
\hline Output Voltage/Freq. & $4.6 \mathrm{kV} / 50 \mathrm{~Hz}$ & $200 \mathrm{~V} / 50 \mathrm{~Hz}$ \\
\hline Switching frequency & $0.7 \mathrm{kHz}$ & $2.5 \mathrm{kHz}$
\end{tabular}

in Fig. 7. Subsequently, the control strategy proposed here was implemented yielding the results presented in Fig. 8 for comparison. In each case the power is constant and the inputport frequency $f_{m}$ is increased in steps every $2 \mathrm{~s}$ from $42 \mathrm{~Hz}$ to $50 \mathrm{~Hz}$, as illustrated in Fig.7(a) and Fig. 8(a). For both cases, the common-mode voltage has an amplitude of $1.25 \mathrm{kV}$. Moreover, for closed-loop EFM control, the common-mode voltage is defined as in (33), with a 3rd order harmonic method for the circulating currents [24], where $A_{1}=1.473, A_{3}=0.295$, and $f_{n}=120 \mathrm{~Hz}$.

Fig. 6 illustrated the main difference between the two approaches demonstrated in Fig. 7 and Fig. 8. The previous methods regulate the average value of the $\mathrm{CCV}$ and use feedforward mitigating signals to reduce the voltage oscillations. On the other hand, the proposal here directly regulates the $\mathrm{CCV}$ (including the voltage oscillations) in a $d q$ frame.

For each result, the CCVs are successfully regulated to $14 \mathrm{kV}$, as shown in Fig. 7(b) and Fig. 8(b). The CCV imbalance terms $v_{c_{0 \alpha}}, v_{c_{0 \beta}}, v_{c_{\alpha 0}}, v_{c_{\beta 0}}$ are presented in Fig. 7(b) and Fig. 8(b). The terms $v_{c_{\alpha \alpha}}, v_{c_{\alpha \beta}}, v_{c_{\alpha \beta}}, v_{c_{\beta \beta}}$ are illustrated in Fig. 7(c). The CCV vectors $\boldsymbol{v}_{c_{1 \alpha \beta}}^{\Sigma \Delta}$ and $\boldsymbol{v}_{c_{2 \alpha \beta}}^{\Sigma \Delta}$ are presented in Fig. 8(c), respectively. In both cases, the ripple of the imbalance terms is properly bounded and it is not increased as $f_{m}$ gets closer to $f_{g}$. However, better performance is obtained using the method proposed here. In fact, the eight imbalance terms are bounded inside a $\pm 350 \mathrm{~V}$ band, which represents oscillations of $\approx 4 \%$ of the CCV nominal value. The control systems presented in [15], [17], [18] use Proportional and PI controllers implemented in $\alpha \beta$ components which cannot regulate sinusoidal signals with zero steady-state error. In contrast, the proposed closed-loop EFM control system provides zero steady-state error in the $\mathrm{CCV}$ even when $f_{m}=50 \mathrm{~Hz}$.Fig. 7(d) and Fig. 8(d) show the constant power transfer between the ports.

Finally, the circulating currents using both methods are presented in Fig. 9. Notice that in Fig 9(b), the circulating currents have a fundamental frequency of $120 \mathrm{~Hz}$ (due to the definition of (33)). Additionally, it is observed that the circulating current magnitude is lower using the method proposed in this paper, which will result in less additional power losses.

\section{EXPERIMENTAL RESULTS}

Experimental results for the proposed control methodology have been obtained using a 27-power-cell $M^{3} C$ prototype shown in Fig. 10. The parameters considered are shown in Table I. The prototype is composed of a control platform and a power stage. A Texas Instrument 6713C DSP board, three FPGA boards and additional external boards for computer

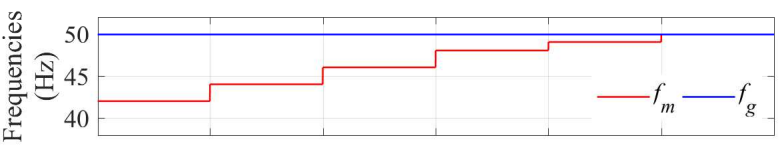

(a)

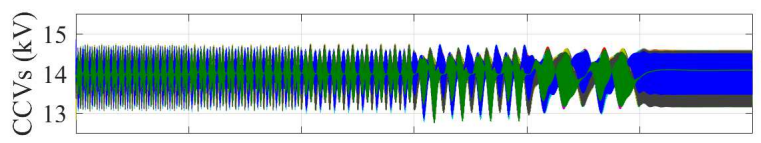

(b)

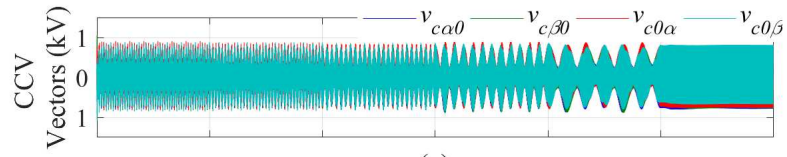

(c)

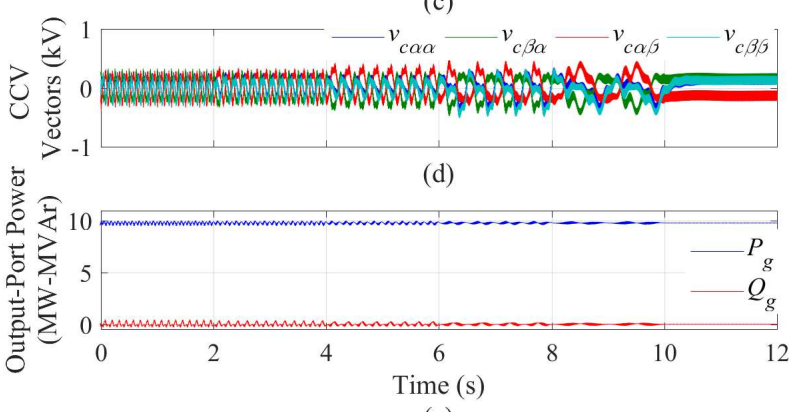

(e)

Fig. 7. Simulation Results for EFM Control as proposed in [17]. (a) input-output ports Frequencies. (b) CCVs. (c) Vectors $\boldsymbol{v}_{c_{0}}^{\alpha \beta}, \boldsymbol{v}_{c_{\alpha \beta}}^{0}$. (d) Components $v_{c_{\alpha \alpha}}, v_{c_{\alpha \beta}}, v_{c_{\alpha \beta} \beta}, v_{c_{\beta \beta}}$. (e) Power injected into the grid.

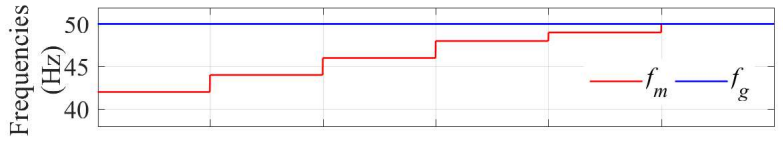

(a)

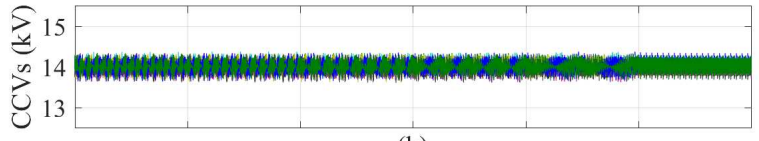

(b)
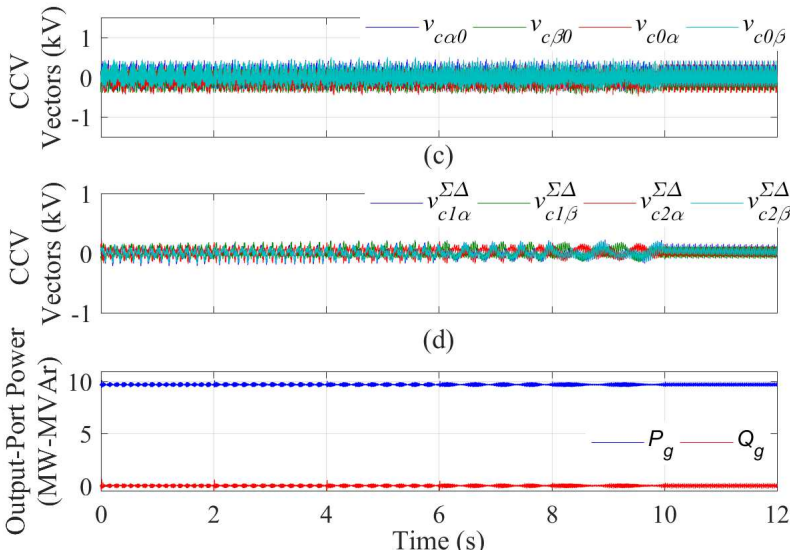

夏

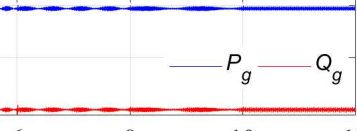

Time $(s)$

(e)

Fig. 8. Simulation Results for the proposed Closed-loop EFM Control System. (a) input-output ports Frequencies. (b) CCVs. (c) Vectors $\boldsymbol{v}_{c_{0}}^{\alpha \beta}$, $\boldsymbol{v}_{c_{\alpha \beta}}^{0}$. (d) Vectors $\boldsymbol{v}_{c_{1 \alpha \beta} \Delta}^{\Sigma \Delta}, \boldsymbol{v}_{c_{2 \alpha \beta}}^{\Sigma \Delta}$. (e) Power injected into the grid.

communication and analogue-digital conversion form the control platform. Additionally, the control platform is equipped with hardware protections to avoid over voltage/currents. The voltage protections are activated when any of the capacitor voltages is higher than $170 \mathrm{~V}$ or when the currents are higher than 20A. The power stage of the prototype is composed of 

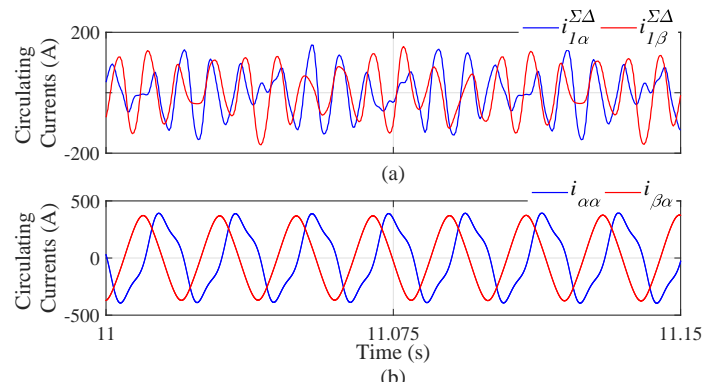

(b)

Fig. 9. Simulation Results. (a) Circulating currents using the method proposed in this paper. (b) Circulating currents using [17].

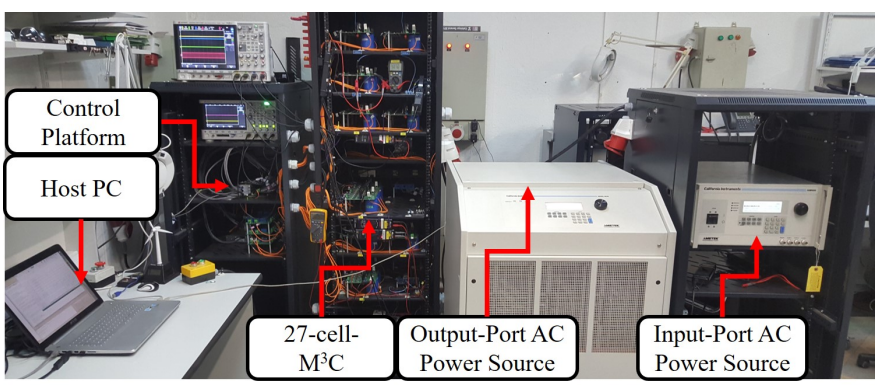

Fig. 10. Downscaled Laboratoy Prototype.

nine clusters, each one based on the series connection of three full-bridge cells and one $2.5 \mathrm{mH}$ inductor. Each cell uses a floating capacitor of $4.7 \mathrm{mF}$. The output-port is connected to an Ametek Programmable power source, Model CSW5550, whereas the output-port is connected to another Ametek Programmable Power Source, Model MX45.

\section{A. Case I: DFM control}

In this case, the DFM control systems for $\boldsymbol{v}_{c_{1 \alpha \beta}}^{\Sigma \Delta}, \boldsymbol{v}_{c_{2 \alpha \beta}}^{\Sigma \Delta}$, $\boldsymbol{v}_{c_{0}}^{\alpha \beta}$ and $\boldsymbol{v}_{c_{\alpha \beta}}^{0}$ are tested. The $M^{3} C$ operates with unity power factor injecting $4 \mathrm{~kW}$ to the input-port. The input/output port voltages are regulated to $200 \mathrm{~V}$ (peak value phase-to-neutral). Experimental results for variable frequency are presented in Fig. 11. As shown in Fig. 11(a), a ramp of frequency between $16 \mathrm{~Hz}$ (the lower limit of the Ametek Power Sources) and $40 \mathrm{~Hz}$ is applied. The 27 capacitor voltages are well regulated to $v_{c}^{*}=150 \mathrm{~V}$, as depicted in Fig. 11(b). The CCV vectors $\boldsymbol{v}_{c_{1 \alpha \beta}}^{\Sigma \Delta}-\boldsymbol{v}_{c_{2 \alpha \beta}}^{\Sigma \Delta}$ and $\boldsymbol{v}_{c_{0}}^{\alpha \beta}-\boldsymbol{v}_{c_{\alpha \beta}}^{0}$ are illustrated in Fig. 11(c) and Fig. 11(d), respectively. The average components of the CCV vectors are $\approx 0$ regardless of $f_{m}$, and the oscillatory components in these vectors are bounded inside a $\pm 7 \mathrm{~V}$ band.

Scope waveforms of the voltages and currents of the $M^{3} C$ are presented in Fig. 11 for $f_{m}=25 \mathrm{~Hz}$. Fig. 11(e) from top to bottom shows: One of the $M^{3} C$ capacitor voltages $v_{c_{a r 1}}$; the cluster voltage $v_{a r}$; and the Output-Input ports voltages $v_{m_{a b}}$ (purple line) and $v_{g_{r t}}$ (blue line). As shown in Fig. 11(f), the output-port currents are controlled to a peak value of $\approx 14 \mathrm{~A}$. Moreover, the port currents are not affected by the circulating currents produced by the DFM control system.

\section{B. Case II: Open-Loop EFM Control}

The $M^{3} C$ is tested for operation at similar input/output port frequencies. The input-port frequency has been set to 48 $\mathrm{Hz}$, whereas the output-port frequency is $50 \mathrm{~Hz}$. As described in Section IV-B, $Q_{m}=-Q_{g}$ and $V_{m}=V_{g}$. The remaining operational set-points are the same as in Case I.

Fig. 12(a) shows that the 27 capacitor voltages are controlled to $v_{c}^{*}=150 \mathrm{~V}$. The operational restrictions over the re-
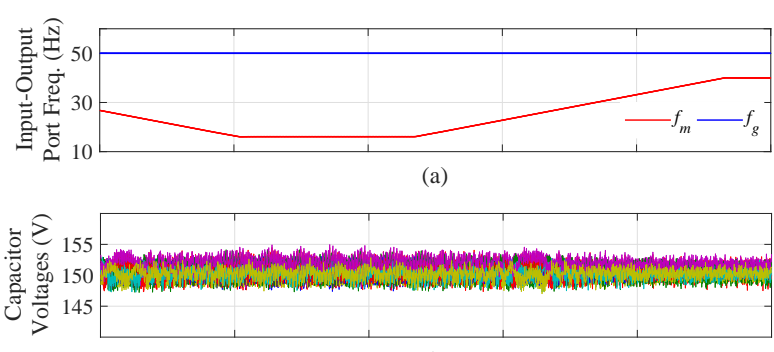

(b)

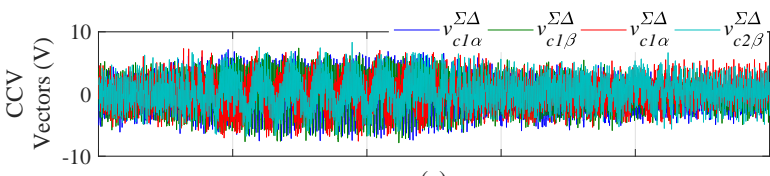

(c)

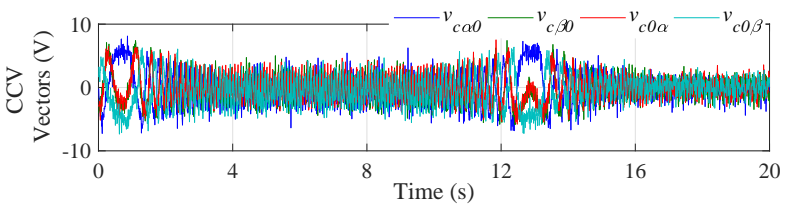

(d)

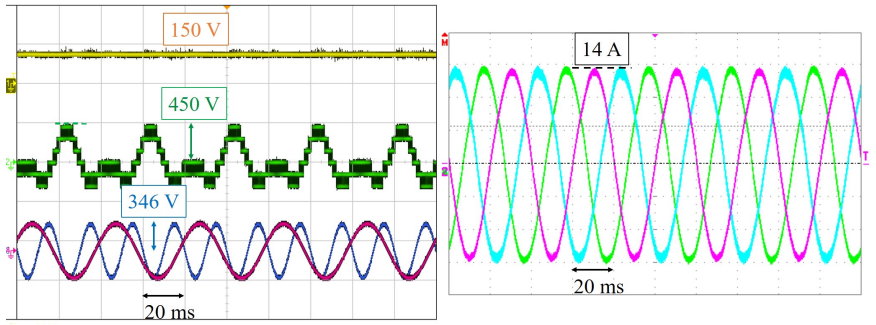

(e)

(f)

Fig. 11. Experimental Results for Case I. (a) input-output ports frequencies. (b) 27 Floating Capacitor Voltages. (c) Vectors $\boldsymbol{v}_{c_{0} \beta}^{\alpha \beta}, \boldsymbol{v}_{c_{\alpha \beta} \beta}^{0}$. (d) Vectors $\boldsymbol{v}_{c_{1 \alpha \beta}}^{\Sigma \Delta}, \boldsymbol{v}_{c_{2 \alpha \beta}}^{\Sigma \Delta}$. (e) Voltages. (f) Input-port currents.

active power and voltage amplitudes, $Q_{m}=-Q_{g}$ and $V_{m}=V_{g}$, mitigate part of the oscillations. Nevertheless, as aforementioned, the values of $Q_{g}$ and $V_{m}$ are manipulated using an open-loop control scheme, where it is difficult to drive the low-frequency oscillating power terms to zero and voltage oscillations still appear on the floating capacitors. Additionally, because of the integral effect of the capacitors, even small lowfrequency power terms can lead to significant voltage imbalances/oscillations [see the denominator in (15)]. As stated in Section IV-C, the voltage oscillations appear in $\boldsymbol{v}_{c_{1 \alpha \beta}}^{\Sigma \Delta}$ (see Fig. 12(b)). However, the CCV vectors $\boldsymbol{v}_{c_{2 \alpha \beta} \Delta}^{\Sigma \Delta}-\boldsymbol{v}_{c_{0}}^{\alpha \beta}-\boldsymbol{v}_{c_{\alpha \beta}}^{0}$ are well regulated as shown in Fig. 12(b)-(c). Scope waveforms are also presented. From top to bottom, Fig. 12(d) illustrates $v_{c_{a r 1}}$ (yellow line), $v_{a r}$ (green line), $v_{m_{a b}}$ (purple line) and $v_{g_{r t}}$ (blue line). Fig. 12(e) illustrates the grid currents which have a peak amplitude of $\approx 14$ A. Fig. 13 presents results for negative sequence operation. The input-port frequency is set to $-48 \mathrm{~Hz}$. In this case, the voltage oscillations appear in $\boldsymbol{v}_{c_{2 \alpha \beta}}^{\Sigma \Delta}$ (see Fig. 13(b)). Whereas, CCV vectors $\boldsymbol{v}_{c_{1 \alpha \beta}}^{\Sigma \Delta}, \boldsymbol{v}_{c_{0}}^{\alpha \beta}$, and $\boldsymbol{v}_{c_{\alpha \beta}}^{0}$ are well regulated with magnitudes close to zero as shown in Fig. 13(b)-(c). Note that using this regulating scheme the power oscillations are not directly regulated, and closed-loop control is not used to compensate for changes in the operating points, incorrect estimation of the reactive powers, etc. Therefore, in the experimental implementation, it was not possible to operate at equal frequencies even though it is theoretically possible. 


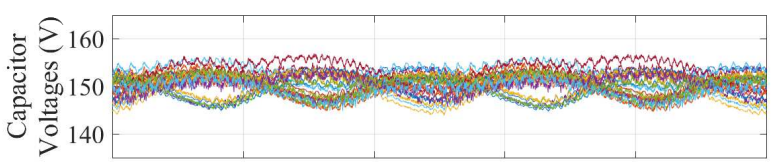

(a)

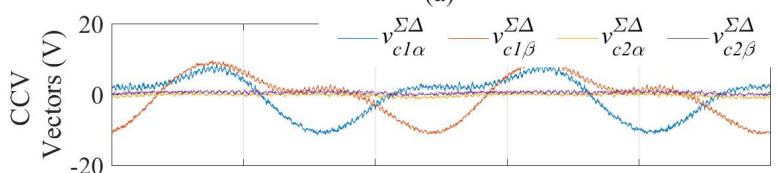

(b)

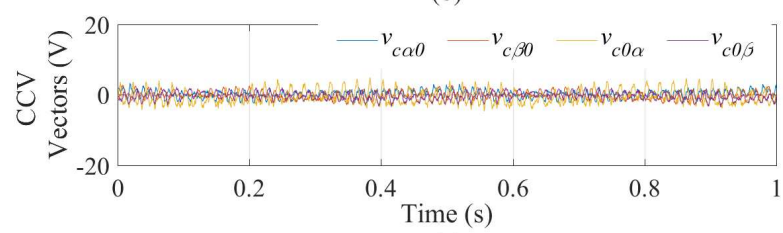

(c)

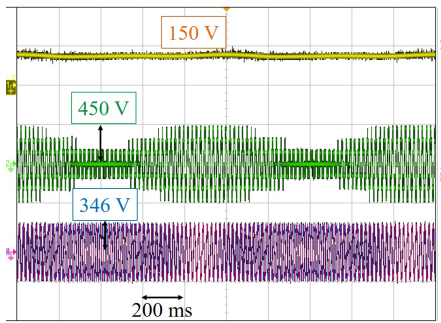

(d)

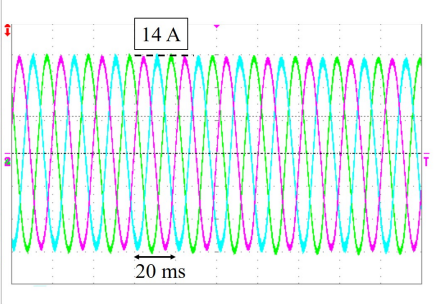

(e)
Fig. 12. Experimental Results for Case II. (a) 27 Floating Capacitor Voltages. (b) Vectors $\boldsymbol{v}_{c_{1 \alpha \beta}}^{\Sigma \Delta}, \boldsymbol{v}_{c_{2 \alpha \beta}}^{\Sigma \Delta}$. (c) Vectors $\boldsymbol{v}_{c_{0}}^{\alpha \beta}, \boldsymbol{v}_{c_{\alpha \beta}}^{0}$. (e) Voltages. (f) Input-port currents.

This is further demonstrated by the experimental results shown in Fig. 13(d), where the performance for a step change from $48 \mathrm{~Hz}$ to $50 \mathrm{~Hz}$ is depicted. In this case, the control system is not able to regulate the capacitor voltage oscillations, and some of the floating capacitor voltages exceed the hardware protection threshold, producing a system shut-down.

\section{Case III: Closed-loop EFM Control}

In this case, the Closed-loop EFM Control of the $M^{3} C$ is enabled. The objective of this test is to validate that the $M^{3} C$ can operate at EFM without operational restrictions. Therefore, both port frequencies are set to $50 \mathrm{~Hz}$, and the converter operates with different power factors. The output-port is set to operate with $P_{m}=-3.3 \mathrm{~kW}$ and $Q_{m}=0 \mathrm{kVAr}$, and the input-port is set with $P_{g}=3.3 \mathrm{~kW}$ and $Q_{m}=2.1 \mathrm{KVAr}$ (to have nominal current amplitude in the input-port). The commonmode voltage is defined as in (33), using a 3rd order harmonic method for the circulating currents [24], where $A_{1}=1.473$, $A_{3}=0.295, V_{0}=30 \mathrm{~V}$ and $f_{n}=120 \mathrm{~Hz}$. As shown in Fig. 14(a), the 27 floating capacitor voltages are well regulated to $v_{c}^{*}=150 \mathrm{~V}$. The vectors $\boldsymbol{v}_{c_{1 \alpha \beta}}^{\Sigma \Delta}$ and $\boldsymbol{v}_{c_{2 \alpha \beta}}^{\Sigma \Delta}$ are illustrated in Fig. 14(b), and $\boldsymbol{v}_{c_{0}}^{\alpha \beta}$ and $\boldsymbol{v}_{c_{\alpha \beta}}^{0}$ are illustrated in Fig. 14(c). In this case, the closed-loop control strategy discussed in Section IV-C, ensures that the CCV vectors are effectively regulated to zero. Additionally, the ripple in these vectors is bounded within $\pm 5 \mathrm{~V}$. Note that in Fig. 15(d), the common-mode voltage waveform is presented. Oscilloscope waveforms are also presented. From top to bottom, Fig. 14(e) illustrates the same variables than those shown in Fig. 11(e), and Fig. 14(f) illustrates the grid currents.

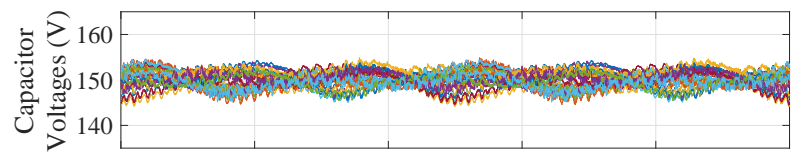

(a)

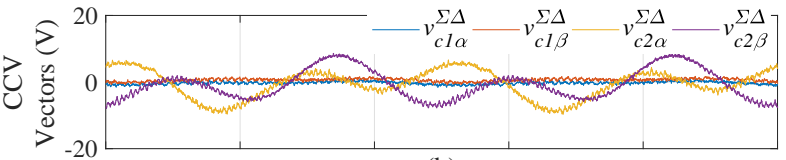

(b)

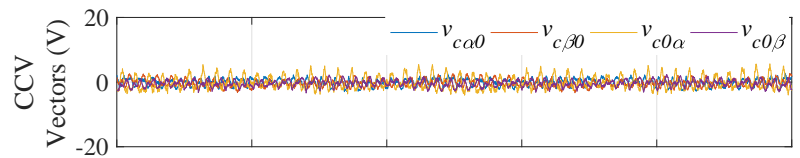

(c)

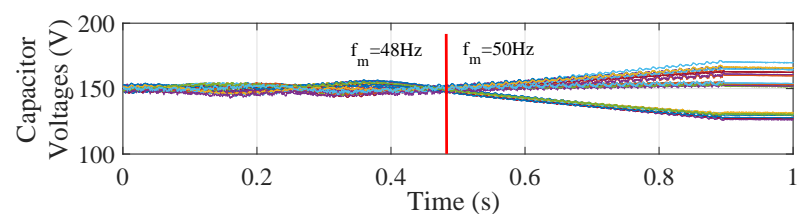

(d)

Fig. 13. Experimental Results for Case II operating with negative sequence. (a) 27 Floating Capacitor Voltages. (b) Vectors $\boldsymbol{v}_{c_{1 \alpha \beta} \Delta}^{\Sigma \Delta}, \boldsymbol{v}_{c_{2 \alpha \beta} \Delta}^{\Sigma \Delta}$. (c) Vectors $\boldsymbol{v}_{c_{0}}^{\alpha \beta}, \boldsymbol{v}_{c_{\alpha \beta}}^{0}$. (d) 27 Floating Capacitor Voltages.

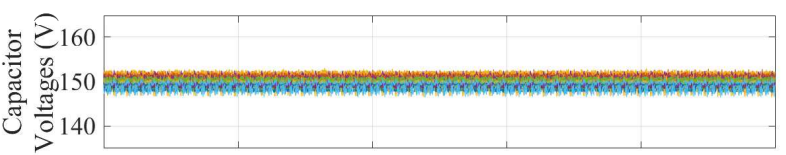

(a)

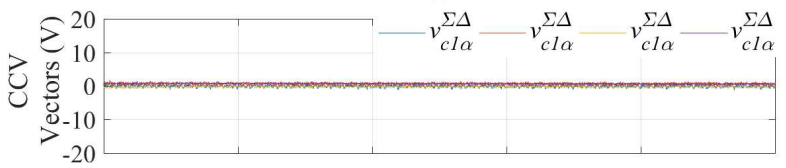

(b)
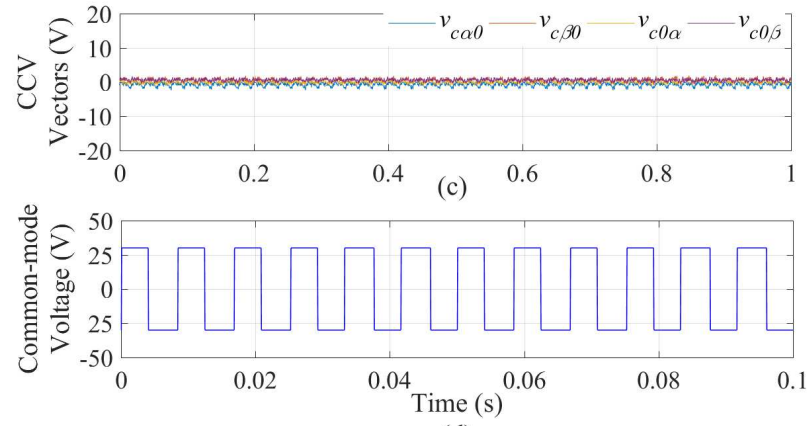

(d)

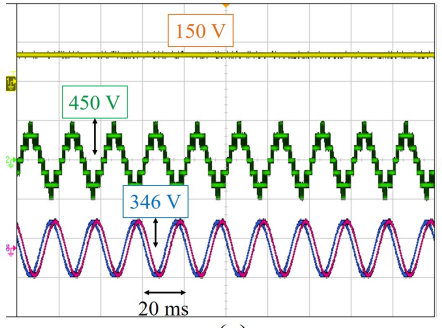

(e)

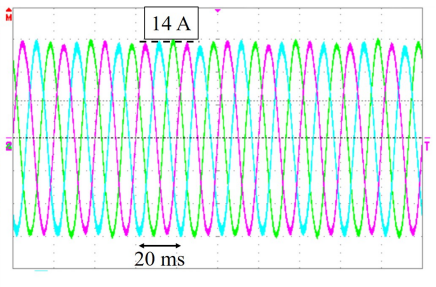

(f)
Fig. 14. Experimental Results for Case III. (a) 27 floating Capacitor Voltages. (b) Vectors $\boldsymbol{v}_{c_{1 \alpha \beta}}^{\Sigma \Delta}, \boldsymbol{v}_{c_{2 \alpha \beta} \Delta}^{\Sigma \Delta}$. (c) Vectors $\boldsymbol{v}_{c_{0}}^{\alpha \beta}, \boldsymbol{v}_{c_{\alpha \beta}}^{0}$. (d) Commonmode voltage. (e) Voltages. (f) Input-port currents. 


\section{CONCLUSIONS}

A new vector control system based on the representation of the $M^{3} C$ converter in $\Sigma \Delta$ double- $\alpha \beta 0$ coordinates has been proposed to enable the operation of the converter over a wide operating range, including equal input/output port frequencies. For mitigation purposes, the vector control system is orientated using synchronous axis systems rotating at $f_{m} \pm f_{g}$.

For the implementation of the control algorithms for EMF operation proposed in this paper, extra voltage/current converter capacity is required for the utilisation of common-mode voltage and circulating currents during EFM. However, the open-loop methods proposed in the literature also require auxiliary signals, and they are also likely to require extra converter ratings. As aforementioned, to obtain good performance with open loop methods during EFM is very difficult and unlikely to be achieved in a practical implementation.

The proposed control algorithms consider two primary operational modes, the DFM and the EFM. The control system for DFM operation regulates the average components of the CCVs using circulating currents. The control system for EFM operation considers the use of circulating currents and common-mode voltage when the difference between the input/output port frequencies is small. It has been shown that the EFM control system performs closed-loop regulation of the CCV vectors driving the low-frequency capacitor voltage oscillations effectively to zero.

Finally, it is highlighted that the vector control system discussed in this work, has been theoretically analysed and experimentally validated using a $27-$ cell- $5 \mathrm{~kW} M^{3} C$ prototype. In the experimental test conditions, decoupled regulation of the input/output ports, balancing of the floating capacitor voltages and mitigation of the oscillations in the floating capacitor voltages have been demonstrated with good performance.

\section{REFERENCES}

[1] R. Marquardt, "Modular Multilevel Converter: An universal concept for HVDC-Networks and extended DC-bus-applications," in 2010 International Power Electronics Conference - ECCE Asia -, IPEC 2010. IEEE, jun 2010, pp. 502-507.

[2] H. Akagi, "Classification, terminology, and application of the modular multilevel cascade converter (MMCC)," IEEE Transactions on Power Electronics, vol. 26, no. 11, pp. 3119-3130, nov 2011.

[3] M. Espinoza, R. Cardenas, M. Diaz, J. J. Clare, R. Cárdenas, M. Díaz, and J. J. Clare, "An Enhanced dq-Based Vector Control System for Modular Multilevel Converters Feeding Variable Speed Drives," IEEE Transactions on Industrial Electronics, vol. 64, no. November, pp. 1-1, 2016.

[4] F. Kammerer, J. Kolb, and M. Braun, "Fully decoupled current control and energy balancing of the Modular Multilevel Matrix Converter," in 15th International Power Electronics and Motion Control Conference and Exposition, EPE-PEMC 2012 ECCE Europe. IEEE, sep 2012, pp. LS2a.3-1-LS2a.3-8.

[5] W. Kawamura, M. Hagiwara, and H. Akagi, "Control and experiment of a modular multilevel cascade converter based on triple-star bridge cells," IEEE Transactions on Industry Applications, vol. 50, no. 5, pp. 3536-3548, sep 2014.

[6] Y. Okazaki, W. Kawamura, M. Hagiwara, H. Akagi, T. Ishida, M. Tsukakoshi, and R. Nakamura, "Which is more suitable for MMCC-based medium-voltage motor drives, a DSCC inverter or a TSBC converter?" in 9th International Conference on Power Electronics - ECCE Asia: "Green World with Power Electronics", ICPE 2015-ECCE Asia. IEEE, jun 2015, pp. 1053-1060.

[7] Y. Okazaki, W. Kawamura, M. Hagiwara, H. Akagi, T. Ishida, M. Tsukakoshi, and R. Nakamura, "Experimental Comparisons Between Modular Multilevel DSCC Inverters and TSBC Converters for Medium-Voltage Motor Drives," IEEE Transactions on Power Electronics, vol. 32, no. 3, pp. 1802-1817, mar 2017.

[8] R. Erickson, S. Angkititrakul, and K. Almazeedi, "A New Family of Multilevel Matrix Converters for Wind Power Applications: Final Report," University of Colorado, Tech. Rep. December, 2006.

[9] M. Díaz, R. Cárdenas, B. Mauricio Espinoza, A. Mora, and F. Rojas, "A novel LVRT control strategy for modular multilevel matrix converter based high-power wind energy conversion systems," in 2015 10th International Conference on Ecological Vehicles and Renewable Energies, EVER 2015. IEEE, mar 2015, pp. 1-11.

[10] M. Diaz, R. Cardenas, M. Espinoza, F. Rojas, A. Mora, J. C. Clare, and P. Wheeler, "Control of Wind Energy Conversion Systems Based on the Modular Multilevel Matrix Converter," IEEE Transactions on Industrial Electronics, vol. 64, no. 11, pp. 1-1, nov 2017.

[11] S. Liu, M. Saeedifard, and X. Wang, "Analysis and Control of the Modular Multilevel Matrix Converter under Unbalanced Grid Conditions," IEEE Journal of Emerging and Selected Topics in Power Electronics, pp. 1-1, 2018.

[12] J. Liu, W. Yao, Z. Lu, and J. Ma, "Design and Implementation of a Distributed Control Structure for Modular Multilevel Matrix Converter," in 2018 IEEE Applied Power Electronics Conference and Exposition (APEC), no. 51677168. IEEE, mar 2018, pp. 1934-1939.

[13] B. Fan, K. Wang, Z. Zheng, L. Xu, and Y. Li, "Optimized Branch Current Control of Modular Multilevel Matrix Converters under Branch Fault Conditions," IEEE Transactions on Power Electronics, vol. 33, no. 6, pp. 4578-4583, jun 2018.

[14] B. Fan, K. Wang, P. Wheeler, C. Gu, and Y. Li, "A Branch Current Reallocation Based Energy Balancing Strategy for the Modular Multilevel Matrix Converter Operating Around Equal Frequency," IEEE Transactions on Power Electronics, vol. 33, no. 2, pp. 1105-1117, feb 2018.

[15] W. Kawamura, M. Hagiwara, and H. Akagi, "A broad range of frequency control for the modular multilevel cascade converter based on triple-star bridge-cells (MMCC-TSBC)," in 2013 IEEE Energy Conversion Congress and Exposition, ECCE 2013. IEEE, sep 2013, pp. 4014-4021.

[16] W. Kawamura, Y. Chiba, and H. Akagi, "A broad range of speed control of a permanent magnet synchronous motor driven by a modular multilevel TSBC converter," in 2016 IEEE Energy Conversion Congress and Exposition (ECCE), 2016, pp. 1-6.

[17] W. Kawamura, Y. Chiba, M. Hagiwara, and H. Akagi, "Experimental Verification of an Electrical Drive Fed by a Modular Multilevel TSBC Converter When the Motor Frequency Gets Closer or Equal to the Supply Frequency," IEEE Transactions on Industry Applications, vol. 53, no. 3, pp. 2297-2306, may 2017.

[18] F. Kammerer, M. Gommeringer, J. Kolb, and M. Braun, "Energy balancing of the Modular Multilevel Matrix Converter based on a new transformed arm power analysis," in 2014 16th European Conference on Power Electronics and Applications, EPE-ECCE Europe 2014. IEEE, aug 2014, pp. 1-10.

[19] M. Diaz, R. Cardenas, M. Espinoza, A. Mora, F. Rojas, and P. Wheeler, "Closed Loop Vector Control of the Modular Multilevel Matrix Converter for equal frequency operation," in Submitted to SPEC, 2017.

[20] R. Cárdenas and R. Peña, "Sensorless vector control of induction machines for variable-speed wind energy applications," IEEE Transactions on Energy Conversion, vol. 19, no. 1, pp. 196-205, mar 2004.

[21] M. Espinoza, R. Cárdenas, M. Díaz, A. Mora, and D. Soto, "Modelling and control of the modular multilevel converter in back to back configuration for high power induction machine drives," in IECON Proceedings (Industrial Electronics Conference). IEEE, oct 2016, pp. 5046-5051.

[22] J. Kolb, F. Kammerer, M. Gommeringer, and M. Braun, "Cascaded control system of the modular multilevel converter for feeding variablespeed drives," IEEE Transactions on Power Electronics, vol. 30, no. 1, pp. 349-357, jan 2015.

[23] J. Kucka, D. Karwatzki, and A. Mertens, "AC/AC modular multilevel converters in wind energy applications: Design considerations," in 2016 18th European Conference on Power Electronics and Applications (EPE'16 ECCE Europe). IEEE, sep 2016, pp. 1-10.

[24] M. Espinoza, E. Espina, M. Diaz, A. Mora, and R. Cardenas, "Improved control strategy of the modular multilevel converter for high power drive applications in low frequency operation," in 2016 18th European Conference on Power Electronics and Applications (EPE'16 ECCE Europe). IEEE, sep 2016, pp. 1-10. [Online]. Available:

[25] H. Akagi, S. Inoue, and T. Yoshii, "Control and Performance of a Transformerless Cascade PWM STATCOM With Star Configuration," 
IEEE Transactions on Industry Applications, vol. 43, no. 4, pp. 1041-1049, 2007.

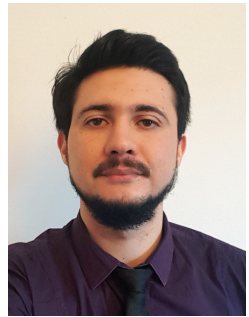

Matias Diaz (S'15) was born in Santiago, Chile. In 2011, he received the B.Sc. and M.Sc. degrees in electrical engineering from the University of Santiago of Chile, Chile. In 2017, he obtained a dual Ph.D. degree at the University of Nottingham, United Kingdom, and at the University of Chile, Chile. From 2013 to 2015, he was the Subdirector of the School of Engineering, Duoc-UC, Chile. Currently, Dr. MatÂas DÃaz is an Assistant Professor at the University of Santiago of Chile. His main research interests include the control of wind energy conversion systems and multilevel converters.

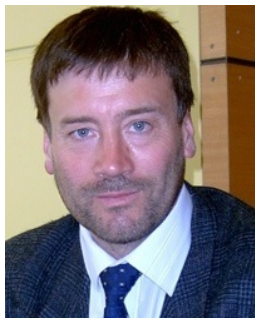

Roberto Cardenas (S'95-M'97-SM'07) was born in Punta Arenas Chile. He received his B.S. degree from the University of Magallanes, Chile, in 1988 and his Msc. and Ph.D degrees from the University of Nottingham in 1992 and 1996 respectively. From 1989-1991 and 1996-2008 he was a lecturer in the University of Magallanes Chile. From 1991 to 1996 he was with the Power Electronics Machines and Control Group (PEMC group), University of Nottingham, United Kingdom. From 2009-2011 he was with the Electrical Engineering Department, University of Santiago. He is currently a Professor of power electronics and drives in the Electrical Engineering Department, University of Chile, Chile. His main interests are in control of electrical machines, variable speed drives and renewable energy systems.

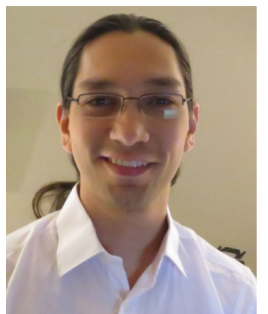

Mauricio Espinoza-B. (S'15) was born in Alajuela, Costa Rica. He received the B.S. and Lic. degrees in electrical engineering from the University of Costa Rica in 2010 and 2012 respectively. From 2010-2014 he was a lecturer in the University of Costa Rica. Actually, he is pursuing a Ph.D. degree at the University of Chile, Chile. Currently, Mauricio Espinoza is an Assistant Professor at the University of Costa Rica. During his career, he has worked in research projects related to modular multilevel converters, machine modelling and control systems for power electronics.

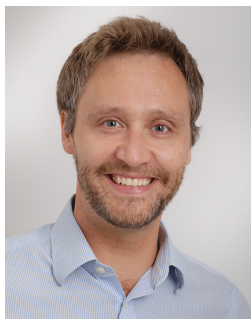

Christoph M. Hackl (M'12-SM'16) was born in 1977 in Mannheim, Germany. After studying electrical engineering (controls and mechatronics) at Technical University of Munich (TUM, Germany) and University of Wisconsin-Madison (USA), he received the B.Sc., Dipl.-Ing., and Dr.Ing. (Ph.D.) degree in 2003, 2004 and 2012 from TUM, respectively. Since 2004, he is teaching electrical drives, power electronics, and mechatronic \& renewable energy systems. Since 2014, he is heading the research group "Control of Renewable Energy Systems (CRES)" at TUM. In February 2018, he has been appointed Professor for Electrical Machines and Drives at the Munich University of Applied Sciences (MUAS), Germany. His main research interests are nonlinear, adaptive and optimal control of electric, mechatronic and renewable energy systems.

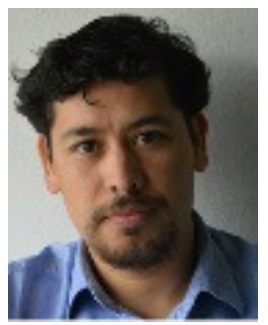

Felix Rojas (M'12-SM'16) Felix Rojas was born in Santiago, Chile. He received the B.Eng. and M.Sc. degrees in electrical engineering in 2009 , from the Universidad de Santiago de Chile, Chile. In 2015, he obtained his doctoral degree from the Technical University of Munich, Germany. During his career, he has worked in research projects related to active filters, matrix converters and multilevel converters. Currently Dr. Rojas work as Assistant Professor at the University of Santiago, Chile. His main research interest are modular multilevel converters for distribution networks and electrical drives.

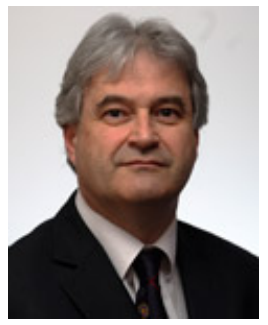

Jon Clare (M'90-SM'04) was born in Bristol, U.K., in 1957. He received the B.Sc. and Ph.D. degrees in electrical engineering from the University of Bristol, Bristol. From 1984 to 1990, he was a Research Assistant and Lecturer with the University of Bristol, where he was involved in teaching and research on power electronic systems. Since 1990, he has been with the Power Electronics, Machines and Control Group, The University of Nottingham, Nottingham, U.K., where he is currently a Professor of power electronics. Prof Clare holds a Royal Society Wolfson Research Merit award. His research interests include power-electronic converters and modulation strategies, variable-speed-drive systems, and electromagnetic compatibility.

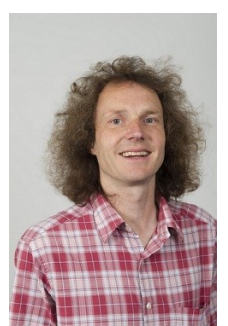

Pat Wheeler (M'90-SM'04) received his BEng [Hons] degree in 1990 from the University of Bristol, UK. He received his PhD degree in Electrical Engineering for his work on Matrix Converters from the University of Bristol, UK in 1994. In 1993 he moved to the University of Nottingham and worked as a research assistant in the Department of Electrical and Electronic Engineering. In 1996 he became a Lecturer in the Power Electronics, Machines and Control Group at the University of Nottingham, UK. Since January 2008 he has been a Full Professor in the same research group. He was Head of the Department of Electrical and Electronic Engineering at the University of Nottingham from 2015 to 2018 . He is currently the Head of the Power Electronics, Machines and Control Research Group and is the Li Dak Sum Chair Professor in Electrical and Aerospace Engineering at the University of Nottingham, China. He is a member of the IEEE PELs AdCom and was an IEEE PELs Distinguished Lecturer from 2013 to 2017. He has published 500 academic publications in leading international conferences and journals. 